\title{
Coherence approach in neutron, x-ray, and neutron spin-echo reflectometry
}

\author{
Victor O. de Haan, * Jeroen Plomp, M. Theo Rekveldt, and Ad A. van Well \\ Department Radiation, Radionuclides \& Reactors, Faculty of Applied Sciences, Delft University of Technology, \\ Mekelweg 15, 2629 JB Delft, The Netherlands \\ Robert M. Dalgliesh and Sean Langridge \\ Rutherford Appleton Laboratory, Chilton, Oxfordshire OX11 OQX, United Kingdom
}

Amarante J. Böttger and Ruud Hendrikx

Department Materials Science and Engineering, Faculty of Mechanical, Maritime and Materials Engineering, Delft University of Technology, Mekelweg 2, 2628 CD Delft, The Netherlands

(Received 15 December 2009; revised manuscript received 1 March 2010; published 23 March 2010)

\begin{abstract}
Based on the application of coherence theory to neutron scattering a description is given of the propagation of neutrons or x-rays through a reflectometer. Important coherence effects at the sample position are discussed. Further, an outline is given how to determine the measured count rate in a detector on the basis of this method including neutron-polarization effects. It is shown in what way the Born approximation and distorted-wave Born approximation can be used within this theory. An outline is given of the phase-object approximation, describing the specular and diffuse scattering from a surface with large surface structure, extending over the existing capabilities of the distorted-wave Born approximation. The incorporation of neutron-polarization effects enables the detailed discussion of neutron spin-echo coding techniques applied to reflectometry.
\end{abstract}

DOI: 10.1103/PhysRevB.81.094112

\section{INTRODUCTION}

The coherence theory was advanced for neutron scattering by Gähler et al. ${ }^{1}$ They showed that coherence theory can be used to describe the propagation of neutrons through instruments. Rauch et al. ${ }^{2}$ and Pushin et al. ${ }^{3}$ applied coherence theory for their measurements with Bonze-Hart interferometers. Moreover, in a recent paper $^{4}$ it was shown that by using the coherence matrix formalism it is possible to describe the propagation of both up- and down-spin states of a neutron wave. The principles of this method and the application of coherence theory to the propagation of neutrons through a reflectometer are described in the following section. As the reflectivity of $\mathrm{x}$-rays can be described in a similar way, the description of the propagation of nonpolarized neutrons also holds for x-rays. Further, the development of spin-echo angular labeling techniques successfully applied in spin-echo small-angle neutron scattering ${ }^{5,6}$ can be used to code the off-specular neutron scattering, which in turn yields information about in-plane inhomogeneities. In contrast to the conventional off-specular methods, where experiments are performed in reciprocal space, the spin-echo method probes directly in real space (on length scales from approximately $20 \mathrm{~nm}-20 \mu \mathrm{m})$. The main object of this paper is the theoretical description and the physical interpretation of the specular and diffuse scattering in reciprocal space as obtained by neutron or x-ray scattering theory and in real space as obtained by the neutron spin-echo technique. The theoretical frame work will be described in terms of wave functions. For the x-ray case a completely analog derivation can be made by changing the wave function into an appropriate electric or magnetic field quantity. ${ }^{7}$

\section{COHERENCE THEORY}

Coherence theory describes the correlation of a field at different locations in space and at different times. For an
PACS number(s): 61.05.fj, 42.25.Kb, 68.49.-h, 42.25.Fx

accurate description the reader can consult Ref. 8. The field considered here is the neutron-wave function. Let $\Psi(\vec{r}, t)$ denote a neutron-wave function characterizing the field at point $\vec{r}$ at time $t$. For a realistic neutron source it will be a fluctuating function of time and may be regarded as a typical member of an ensemble consisting of all possible neutrongenerating events. It consists of a large number of Fourier components which are independent of each other so that their superposition gives rise to a fluctuating field which is only describable in statistical terms. For a statistically stationary beam it can be constructed from its constituting monochromatic waves ${ }^{9}$

$$
\Psi(\vec{r}, t)=\int \psi_{\vec{k}}(\vec{r}) e^{-i \omega_{k} t} d^{3} k,
$$

where $\vec{k}$ is the wave vector or propagation vector, $\omega_{k}=v_{p} k$ where $v_{p}=\hbar k / 2 m$ equals the phase velocity of the wave function, half of the neutron velocity, and $m$ equals the neutron mass. For x-rays the phase velocity in vacuum is equal to the speed of light. We will use the formalism of the coherence matrix, $\hat{\Gamma}$ as developed in Ref. 4 to describe the propagation of the polarized neutron beam trough the instrument. This will be discussed in Sec. IV. For nonpolarized neutrons the formalism reduces to the propagation of the mutual coherence function, $\Gamma$. This is used in Sec. III. Further definitions of the parameters are equal to those in Ref. 4.

\section{REFLECTOMETRY}

For reflectometers the typical instrument geometry is shown in Fig. 1. A neutron created by a source $x=x_{0}$, represented by a mutual coherence function $\Gamma_{0}$ propagates through space toward the sample position $x=x_{s}$. At the sample position the incident neutron is represented by the mutual coherence function $\Gamma_{i n}$ and interacts with the sample. After the 


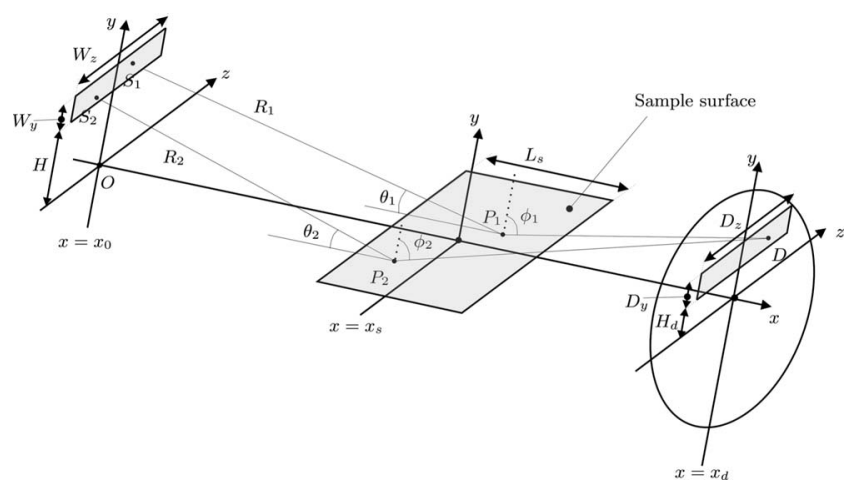

FIG. 1. Notation relating to the propagation of the mutual coherence function through a reflectometer.

interaction the scattered neutron is represented by the mutual coherence function, $\Gamma_{s c}{ }^{1}$

\section{A. Propagation from source to sample}

For a completely homogeneous incoherent source with area $A_{0}$ the mutual coherence function at the source position is given by Eq. (31) of Ref. 4. Substituting this in Eq. (30) of Ref. 4 yields for the mutual coherence function at the sample position

$$
\Gamma_{i n}\left(\vec{r}_{1}, \vec{r}_{2}, \tau\right)=\frac{J_{0}}{2 v_{p}} \frac{e^{-i k v_{p} \tau}}{4 \pi} \int_{A_{0}} \frac{e^{i k\left(R_{2}-R_{1}\right)}}{R_{2} R_{1}} d^{2} r_{1}^{\prime},
$$

where for reflectometry in the far-zone $\cos \theta_{i} \approx 1$. If $\vec{r}_{2}=\vec{r}_{1}$ $+\vec{r}, \vec{r}=(\Delta x, \Delta y, \Delta z)^{\mathrm{T}}$, and $r \ll r_{1}$ than $R_{2}-R_{1}$ can be approximated by
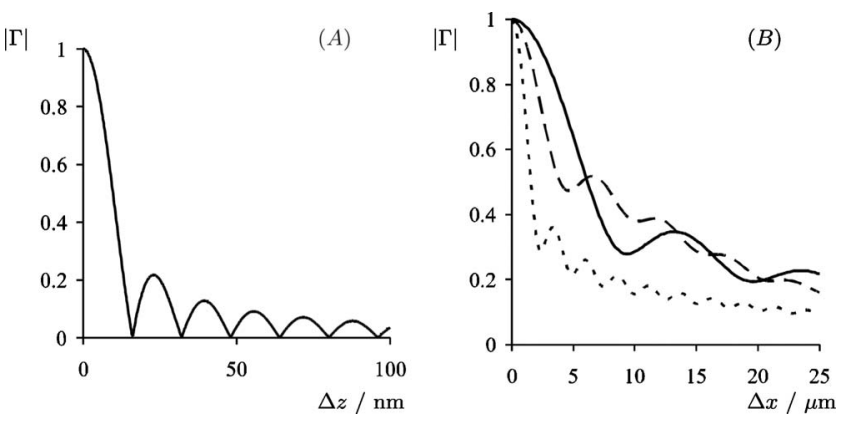

FIG. 2. (A) Amplitude of the normalized mutual coherence function, $\Gamma$ cut along the $\Delta z$ axis $(\Delta x=0)$ for $z_{1}=0,1$, and $2.5 \mathrm{~cm}$ (all coincide on black line). ( $B)$ The same function cut along the $\Delta x$ axis $(\Delta z=0)$ at the sample surface for $z_{1}=0$ (full line), $z_{1}=1 \mathrm{~cm}$ (long-dashed line), and $z_{1}=2.5 \mathrm{~cm}$ (short-dashed line). The instrument parameters used are given in Table I.

$$
R_{2}-R_{1} \approx-\Delta x\left(1-\zeta_{z}^{2} / 2-\zeta_{y}^{2} / 2\right)-\Delta y \zeta_{y}-\Delta z \zeta_{z}
$$

where $\zeta_{z}=\left(\overrightarrow{r_{1}^{\prime}}-\vec{r}_{1}\right) \cdot \vec{e}_{z} / r_{1}$ and $\zeta_{y}=\left(\overrightarrow{r_{1}^{\prime}}-\vec{r}_{1}\right) \cdot \vec{e}_{y} / r_{1}$. The denominator in the integral of Eq. (2) can be reduced to $r_{1}^{2}$ without introducing a large error. If the source aperture is rectangular with a height $W_{y}$ and a width $W_{z}$ and the middle of the aperture is situated at $y=H$ and $z=0$ the integral can be evaluated as

$$
\begin{aligned}
\Gamma_{i n}\left(\vec{r}_{1}, \vec{r}_{1}+\vec{r}, \tau\right) \approx & \frac{J_{0}}{2 v_{p}} \frac{e^{i k\left(\Delta x-v_{p} \tau\right)}}{4 \pi r_{1}^{2}} e^{i \pi / 2\left(\kappa_{y} / \kappa_{x}\right)^{2}} \frac{E\left[\kappa_{x}\left(\kappa_{y} / \kappa_{x}^{2}+H-y_{1}+W_{y} / 2\right)\right]-E\left[\kappa_{x}\left(\kappa_{y} / \kappa_{x}^{2}+H-y_{1}-W_{y} / 2\right)\right]}{\kappa_{x}} \\
& \times e^{i \pi / 2\left(\kappa_{z} / \kappa_{x}\right)^{2}} \frac{E\left[\kappa_{x}\left(\kappa_{z} / \kappa_{x}^{2}-z_{1}+W_{z} / 2\right)\right]-E\left[\kappa_{x}\left(\kappa_{z} / \kappa_{x}^{2}-z_{1}-W_{z} / 2\right)\right]}{\kappa_{x}}
\end{aligned}
$$

with

$$
E(x)=C(x)+i S(x)
$$

where $C(x)$ and $S(x)$ are the cosine and sine Fresnel integrals $^{10}$ and $\kappa_{x}^{2}=k \frac{\Delta x}{\pi r_{1}^{2}}, \kappa_{y}=\frac{k \Delta y}{\pi r_{1}}$, and $\kappa_{z}=\frac{k \Delta z}{\pi r_{1}}$. Note that $\lim _{x \rightarrow \infty} E(x)=(1+i) / 2-i \exp \left(-i \pi x^{2} / 2\right) / \pi x+O\left(x^{-3}\right)$. This limit can be used to find the mutual coherence function for $|\Delta x| \ll \Delta y^{2} k, \Delta z^{2} k$

$$
\begin{aligned}
& \Gamma_{i n}\left(\vec{r}_{1}, \vec{r}_{1}+\vec{r}, \tau\right) \\
& \approx \frac{J_{0}}{2 v_{p}} \frac{W_{y} W_{z} e^{i k\left(\Delta x-v_{p} \tau\right)}}{4 \pi r_{1}^{2}} \frac{\sin \left(\frac{\pi}{2} \kappa_{y} W_{y}\right)}{\frac{\pi}{2} \kappa_{y} W_{y}} \frac{\sin \left(\frac{\pi}{2} \kappa_{z} W_{z}\right)}{\frac{\pi}{2} \kappa_{z} W_{z}} .
\end{aligned}
$$

Further, note that $\lim _{x \rightarrow 0} E(x)=x\left[1+O\left(x^{4}\right)\right]-i x^{3}[\pi / 6$
$\left.+O\left(x^{4}\right)\right]$. This limit can be used to find the neutron density at the sample surface

$$
\Gamma_{i n}(\vec{r}, \vec{r}, 0)=\frac{J_{0}}{2 v_{p}} \frac{W_{z} W_{y}}{4 \pi r_{1}^{2}} .
$$

Examples of the amplitude of the normalized mutual coherence function are shown in Fig. 2. The parameters used in the calculations are given in Table I. Figure A shows the function along the $\Delta z$ direction $(\Delta x=0)$. There is no strong dependence on $z_{1}$ nor on $x_{1}$. Figure $\mathrm{B}$ shows the function along the $\Delta x$ direction $(\Delta z=0)$ at the sample surface for several positions on the sample $z_{1}=0 \mathrm{~cm}, z_{1}=1 \mathrm{~cm}$, and $z_{1}$ $=2.5 \mathrm{~cm}$. Note the difference in scale for the $\Delta x$ and $\Delta z$ directions. This is due to the difference in diaphragm width and due to the small glancing angles $\theta_{i}$. The width of the mutual coherence function along the $\Delta x$ direction reduces when the distance to the center of the sample increases. This is due to a small rotation $z_{1} / r_{1}$ of the mutual coherence func- 
TABLE I. Parameters for calculation of examples of properties of a reflectometer.

\begin{tabular}{lccc}
\hline \hline Symbol & Description & Quantity & Unit \\
\hline$H$ & Height diaphragm above sample surface & 4 & $\mathrm{~cm}$ \\
$W_{y}$ & Width diaphragm in $y$ direction & 1 & $\mathrm{~mm}$ \\
$W_{z}$ & Width diaphragm in $z$ direction & 5 & $\mathrm{~cm}$ \\
$r_{1}$ & Distance between diaphragm and sample & 4 & $\mathrm{~m}$ \\
$H_{d}$ & Height detector diaphragm above sample surface & 4 & $\mathrm{~cm}$ \\
$D_{y}$ & Width detector diaphragm in $y$ direction & 1 & $\mathrm{~mm}$ \\
$D_{z}$ & Width detector diaphragm in $z$ direction & 5 & $\mathrm{~cm}$ \\
$r_{d s}$ & Distance between sample and detector & 1 & $\mathrm{~m}$ \\
$\lambda$ & Wavelength used in calculations & 0.2 & $\mathrm{~nm}$ \\
\hline \hline
\end{tabular}

tion around the $y$ axis, originating from a slightly different direction of the beam incident on the sample. To elucidate this, a two-dimensional plot of the mutual coherence function along the sample surface as function of both $\Delta x$ and $\Delta z$ at the middle of the sample $\left(z_{1}=0\right)$ is shown in Fig. 3. Note again the difference in scales in the $\Delta x$ and $\Delta z$ directions. Interesting fact is that the mutual coherence function has an extended tail in the direction $|\Delta z| \approx\left|\Delta x\left(W_{z} / 4+z_{1}\right) / r_{1}\right|$. This shows that if the coherence properties of the beam are split into two separate contributions for the $\Delta x$ and $\Delta z$ directions, the coherence of the beam might be under or over estimated. Further, the coherence properties of the incident beam strongly depend on the position on the sample in the direction perpendicular to the beam. The coherence properties vary much less as function of the position on the sample in the direction parallel to the beam.

\section{B. Propagation from sample to detector}

The count rate in the detector is due to the scattered mutual coherence matrix propagated from the sample position to the detector and can be found by an integral of the neutron flux $\vec{J}\left(\vec{r}_{d}\right)=2 \vec{v}_{p} \rho\left(\vec{r}_{d}\right)$ over the detector area, $A_{d}$ at a position $\vec{r}_{d}$, according to Eq. (40) of Ref. 4

$$
I_{d}=2 v_{p} \int_{A_{d}} \Gamma\left(\vec{r}_{d}, \vec{r}_{d}, 0\right) d^{2} r_{d},
$$

where it was assumed that the detector area is perpendicular to the beam direction. Equation (30) of Ref. 4 is used to calculate the propagation to the detector when $R_{i}=\left|\vec{r}_{d}-\vec{r}_{i}\right|$ is taken. For the integration area the sample plane is taken $(y$ $=0)$ and $\cos \phi_{i}=y_{d} / R_{i}$, where $\left(x_{d}, y_{d}, z_{d}\right)^{\mathrm{T}}=\vec{r}_{d}$. If, again, $\vec{r}_{2}$ $=\vec{r}_{1}+\vec{r}, \vec{r}=(\Delta x, 0, \Delta z)^{\mathrm{T}}$, and $r \ll R_{1}$ the result is

$$
\Gamma\left(\vec{r}_{d}, \vec{r}_{d}, 0\right)=\int_{y=0} \frac{\cos ^{2} \phi_{1}}{R_{1}^{2} \lambda^{2}} \int_{y=0} e^{-i \vec{q}_{d 1} \cdot \vec{r}} \Gamma_{s c}\left(\vec{r}_{1}, \vec{r}_{1}+\vec{r}, 0\right) d^{2} r d^{2} r_{1},
$$

where $\vec{q}_{d 1}=k\left(\vec{r}_{d}-\vec{r}_{1}\right) / R_{1}$. The inner integral of this formula can be interpreted as a Fourier transform of the mutual coherence function at position $\vec{r}_{1}$ on the sample plane. The outer integral is an averaging of that Fourier transform over the whole sample. In the far-zone approximation the angles $\phi_{i}$ are almost $\pi / 2$ radians (see Fig. 1) and $R_{i}$ in the cosine factors and the denominator inside the integral can be taken constant: $R_{i} \approx r_{d s}$ and $\cos \phi_{1}=y_{d} / r_{d s}$. If the detector is located at the sample's horizon (i.e., $y_{d}=0$ ) the neutron density due to the scattered beam becomes 0 . The explanation for
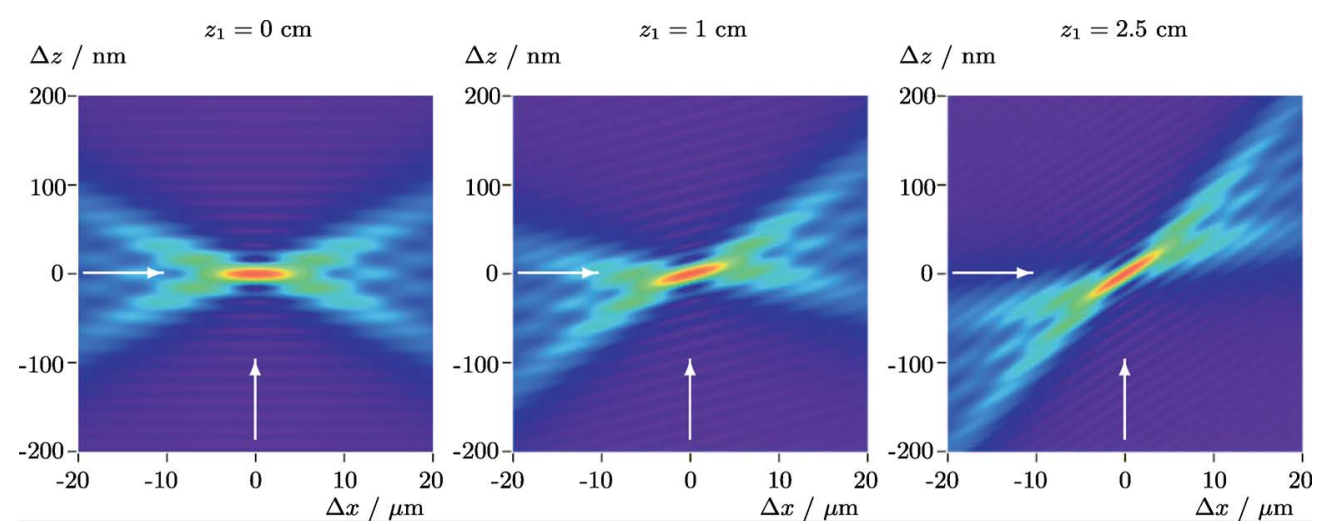

FIG. 3. (Color online) Amplitude of the normalized mutual coherence function at the sample surface for different position on the sample $z_{1}=0 \mathrm{~cm}$ (left), $z_{1}=1 \mathrm{~cm}$ (mid), and $z_{1}=2.5 \mathrm{~cm}$ (right). The instrument parameters used are given in Table I. Note the different scales for the axes. For negative $z_{1}$ the function is mirrored in the $\Delta z=0$ axis with respect to the function for a positive value of $z_{1}$. The white arrows indicate the directions of the cuts of Fig. 2. The color scale is linear ranging from 1 in the middle of the graphs (red) to 0 at the outer limits of the graphs (blue). 
this is that the sample area as seen by a detector at this position is zero. If the detector area is large and the scattering is in the specular direction so that $y_{d} / r_{d s}$ can be taken equal to $y_{1} / r_{1}$, the total count rate in the detector is given by

$$
I_{d}=2 v_{p} \int_{y=0} \frac{y_{1}^{2}}{r_{1}^{2}} \Gamma_{s c}\left(\vec{r}_{1}, \vec{r}_{1}, 0\right) d^{2} r_{1} .
$$

If the scattered mutual coherence function is sufficiently narrow $\vec{q}_{d 1} \cdot \vec{r}$ can be approximated by

$$
\vec{q}_{d 1} \cdot \vec{r} \approx k \Delta x-k \Delta x \frac{y_{d}^{2}+\left(z_{d}-z_{1}\right)^{2}}{2\left(x_{d}-x_{1}\right)^{2}}-k \Delta z \frac{z_{d}-z_{1}}{x_{d}-x_{1}} .
$$

If further, the second and third term can be neglected $(\ll 1)$, the above integral Eq. (9) reduces to a Fourier transform of the $x$ direction of the mutual coherence function. The maximum value of the second or third term is given by the maximum of $k \Delta x$ or $k \Delta z$ and the maximum of either $y_{d} /\left(x_{d}-x_{1}\right)$ or $\left(z_{d}-z_{1}\right) /\left(x_{d}-x_{1}\right)$. The position of the detector should be approximately in the position of the reflected beam. This limits the maximum values of $y_{d} /\left(x_{d}-x_{1}\right)$ and $\left(z_{d}-z_{1}\right) /\left(x_{d}\right.$ $\left.-x_{1}\right)$. The maxima for $\Delta x$ and $\Delta z$ are determined by the spatial resolution of the reflectometer under consideration. If for the maximum of $\Delta x$ the first maximum in Fig. 2(B) is taken and for $\Delta z$ the first 0 of Eq. (6) is taken, the maximum of the second and third term is on the order of 1 . Hence, if the sample correlations extend over much smaller distances than the resolution of the reflectometer as determined by the entrance slit and the sample size, one can safely interpret Eq. (9) as mentioned. However, for sample correlations extending toward and over the resolution of the reflectometer the above complete integral equation should be used to calculate the detector count rate.

For the interpretation of reflectivity measurements the transition at the sample surface from the incident mutual coherence function to the scattered one is needed. This transition cannot be described by means of the coherence theory as the wave function itself is scattered by the optical potential of the scattering object. Hence, to make the transition first it must be known how the scattered wave function is related to the incident wave function. Then, the definition of the mutual coherence function can be applied to determine the transition of the incident mutual coherence function into the scattered one.

\section{Specular reflection}

If the optical potential $V_{r}(\vec{r})$ of the sample is statistically stationary and only a function of the direction perpendicular to the sample surface (here the $y$ direction) the threedimensional time-dependent Schrödinger equation can be reduced to three one-dimensional time-independent equations. The first two differential equations are linear second-order differential equations in $x$ and $z$ and can easily be solved. The last differential equation depends on the potential $V_{r}(y)$. In the region $y>0$ above the sample the potential is 0 and this equation also reduces to a linear second-order differential equation with a general solution ${ }^{11}$

$$
\psi_{k_{y}}(y)=e^{-i k_{y} y}+\rho e^{i k_{y} y} .
$$

The first term at the right-hand side corresponds to the incident beam and the second term to the (specularly) reflected beam, where $\rho$ is the reflectance. A quasimonochromatic beam at the sample surface can be thought of as an ensemble average of plane waves. The scattered mutual coherence function can be calculated by realizing that the propagation formula for the mutual coherence function [Eq. (30) of Ref. 4] consists of two integrals. One integrating the incident wave function reaching point $\vec{r}_{1}$ from $\vec{r}_{1}^{\prime}$ and the other integrating the incident wave function reaching point $\vec{r}_{2}$ from $\vec{r}_{2}^{\prime}$. To find the scattered mutual coherence function one has to multiply (before integrating) the incident wave functions by the appropriate reflectance. For a completely homogeneous incoherent source the scattered mutual coherence function at the sample surface reduces to

$$
\begin{aligned}
\Gamma_{s c}\left(\vec{r}_{1}, \vec{r}_{2}, \tau\right)= & \frac{J_{0}}{2 v_{p}} \frac{e^{-i k v_{p} \tau}}{4 \pi} \\
& \times \int_{A_{0}} \rho\left(q_{1}\right)^{*} \rho\left(q_{2}\right) \frac{\cos \theta_{1} \cos \theta_{2} e^{i k\left(R_{2}-R_{1}\right)}}{R_{2} R_{1}} d^{2} r_{1}^{\prime},
\end{aligned}
$$

where $q_{i}=k\left(\overrightarrow{r_{i}^{\prime}} \cdot \vec{e}_{y}\right) / R_{i}$. If the mutual coherence function is sufficiently narrow $q_{i} \approx q_{1}$ so that $\rho\left(q_{1}\right)^{*} \rho\left(q_{2}\right)=R\left(q_{1}\right)$, the reflectivity. If the source aperture is rectangular the integral can be evaluated as

$$
\Gamma_{s c}\left(\vec{r}_{1}, \vec{r}_{1}+\vec{r}, \tau\right)=\widetilde{R}(q, \Delta q, \alpha) \Gamma_{i n}\left(\vec{r}_{1}, \vec{r}_{1}+\vec{r}, \tau\right),
$$

where $q=k H / r_{1}, \Delta q=k W_{y} / 2 r_{1}, \alpha=\sqrt{\Delta x / \pi k}$, and

$$
\tilde{R}(q, \Delta q, \alpha)=\frac{\int_{q-\Delta q}^{q+\Delta q} R(\eta) e^{-i(\pi / 2) \alpha^{2} \eta^{2}} d \eta}{\int_{q-\Delta q}^{q+\Delta q} e^{-i(\pi / 2) \alpha^{2} \eta^{2}} d \eta}
$$

is a folded reflectivity. Note that for $\Delta x=0$ this reduces to

$$
\tilde{R}(q, \Delta q, 0)=\frac{\int_{q-\Delta q}^{q+\Delta q} R(\eta) d \eta}{2 \Delta q} .
$$

If the scattering (or reflection) is mainly in the specular direction and the detector area is large enough the total count rate in the detector is given by Eq. (10)

$$
I_{d}=2 v_{p} \int_{A_{s}} \frac{q^{2}}{k^{2}} \widetilde{R}(q, \Delta q, 0) \Gamma_{i n}\left(\vec{r}_{1}, \vec{r}_{1}, 0\right) d^{2} r_{1},
$$

which using Eq. (7) reduces for an incoherent source to

$$
I_{d}=J_{0} W_{z} W_{y} \int_{A_{s}} \frac{q^{2}}{k^{2}} \frac{\tilde{R}(q, \Delta q, 0)}{4 \pi r_{1}^{2}} d^{2} r_{1}
$$

and for the slit geometry assumed here 


$$
I_{d}=\frac{J_{0} A_{s} W_{z} W_{y}}{4 \pi r_{1}^{2} k^{2}} \frac{\int_{q-\Delta q}^{q+\Delta q} \eta^{2} R(\eta) d \eta}{2 \Delta q} .
$$

Note that the total reflected beam is detected by the detector and it is assumed that the sample is small so that the resolution is due to the entrance diaphragm and the distance to the sample only.

\section{Scattering}

In case that the mutual coherence function at the sample position can be considered homogeneous, it was shown ${ }^{4}$ that using the coherence theory the count rate at the detector given by Eq. (8) can be expressed as an integral over the sample volume of a product of three factors

$$
I_{d}=2 v_{p} \int R_{\text {out }}\left(\vec{r}_{1}, \vec{r}_{1}+\vec{r}\right) G_{r}(\vec{r}) \Gamma_{i n}\left(\vec{r}_{1}, \vec{r}_{1}+\vec{r}, 0\right) d^{3} r,
$$

where $G_{r}(\vec{r})$ is the sample correlation function determined by the scattering of the neutron-wave function at the scattering object and

$$
R_{\text {out }}\left(\vec{r}_{1}, \vec{r}_{1}+\vec{r}\right)=\int_{A_{d}} \frac{e^{-\vec{p} \cdot \vec{r}}}{\left|\vec{r}_{d}-\vec{r}_{1}\right|^{2}} d^{2} r_{d}
$$

with $\vec{p}=k\left(\vec{r}_{d}-\vec{r}_{1}\right) /\left|\vec{r}_{d}-\vec{r}_{1}\right|$. This function behaves in a similar way as the incoming mutual coherence function at the sample position for a completely incoherent source given by Eq. (4). The relevant dimensions in this case are the distance between sample and detector and the detector pixel position and size. We would like to emphasis that Eq. (20) only holds if the mutual coherence function at the sample position can be taken homogeneous, i.e., independent of $\vec{r}_{1}$. This condition can be relaxed when an average over the sample area is taken. In that case the mutual coherence function needs to be homogeneous over the correlation length of the sample, which can be inferred form the extension of the sample correlation function, $G_{r}$. Then, this equation can be converted into a convolution of an instrumental resolution function and the sample structure factor

$$
I_{d}\left(\vec{r}_{d}\right)=R^{F T}(\vec{Q}) * S_{k}(\vec{Q}),
$$

where $\vec{Q}=\vec{p}-\vec{k}=k\left(\vec{r}_{d}-\vec{r}_{s}\right) /\left|\vec{r}_{d}-\vec{r}_{s}\right|-k\left(\vec{r}_{s}-\vec{r}^{\prime}\right) /\left|\vec{r}_{s}-\vec{r}^{\prime}\right|$ is the wave-vector transfer. The instrumental resolution function is determined by instrument details. For a complete incoherent source

$$
R^{F T}(\vec{Q})=\iint_{A_{0}} \int_{A_{d}} \frac{J_{0} e^{-\vec{Q} \cdot \vec{r}}}{4 \pi\left|\vec{r}_{d}-\vec{r}_{s}\right|^{2}\left|\vec{r}_{s}-\vec{r}^{\prime}\right|^{2}} d^{2} r_{d} d^{2} r^{\prime} d^{3} r .
$$

The sample structure factor is defined as the Fourier transform of the sample correlation function

$$
S_{k}(\vec{Q})=\int e^{-i \vec{Q} \cdot \vec{r}} G_{r}(\vec{r}) d^{3} r .
$$

One can use the Lipmann-Schwinger equation ${ }^{9,12}$ to solve the Schrödinger equation, resulting in the first Born approxi- mation or distorted-wave Born approximation. ${ }^{13-15}$ Another way to solve the scattering of the neutron-wave function is to use the phase-object approximation. ${ }^{4}$

The Lippmann-Schwinger equation can be iterated resulting in an infinite series for $\Psi_{s c}(\vec{r})$ which can be rewritten in the form

$$
\Psi_{s c}(\vec{r})=\Psi_{i n}(\vec{r})-\frac{2 m}{\hbar^{2}} \int G^{(+)}\left(\vec{r}, \vec{r}_{s}\right) V_{r}\left(\vec{r}_{s}\right) \Psi_{i n}\left(\vec{r}_{s}\right) d^{3} r_{s},
$$

where $G^{(+)}\left(\vec{r}, \vec{r}_{s}\right)$ is a specialized (potential-dependent) Green's function describing the scattering into a scatteredwave function and defined by the solution of the following Schrödinger equation

$$
\left[\nabla^{2}+k^{2}-\frac{2 m V_{r}\left(\vec{r}_{s}\right)}{\hbar^{2}}\right] G^{(+)}\left(\vec{r}, \vec{r}_{s}\right)=\delta\left(\vec{r}-\vec{r}_{s}\right),
$$

where the $\nabla^{2}$ operator represents the derivatives to $\vec{r}_{s}$. This equation describes the scattered wave as a superposition of waves produced by many scattering events occurring at different elements of the sample. This Green's function can be approximated by taking the Green's function for the undisturbed potential at position $(x, z)$ along the sample surface. For a layer with a variable height, $H$ on top of a substrate of a different material the Green's function is derived in the Appendix. Inserting Eq. (A17) and the incident-wave function given by

$$
\psi_{i n}(\vec{k}, \vec{r})=\psi_{0}\left(\vec{k}_{\|}\right) e^{i \vec{k} \cdot \vec{r}}
$$

in the scattered-wave function in Eq. (25) yields

$$
\begin{aligned}
\psi_{s c}(\vec{k}, \vec{r})= & \psi_{i n}(\vec{k}, \vec{r})-\psi_{0}\left(\vec{k}_{\|}\right) \int_{0}^{\infty} \frac{e^{i \vec{p} \cdot \vec{r}}}{2 \pi i p_{y}} \\
& \times \int e^{-i \vec{Q} \cdot \vec{r}_{s}} e^{i p_{y} y_{s}} \Psi_{p}\left[y_{s}, H\left(\vec{r}_{s, \|}\right)\right] \frac{m V_{r}\left(\vec{r}_{s}\right)}{2 \pi \hbar^{2}} d^{3} r_{s} d^{2} p_{\|},
\end{aligned}
$$

where again $\vec{Q}$ is the wave-vector transfer defined as $\vec{Q}=\vec{p}$ $-\vec{k}$. The integral over $\vec{r}_{s}$ can be split in an integral over $\vec{r}_{s, \|}$ and $y_{s}$, yielding

$$
\begin{aligned}
\psi_{s c}(\vec{k}, \vec{r})= & \psi_{i n}(\vec{k}, \vec{r})+\psi_{0}\left(\vec{k}_{\|}\right) \\
& \times \int_{0}^{\infty} \frac{e^{i \vec{p} \cdot \vec{r}}}{2 \pi p_{y}} \int e^{-i \vec{Q}_{\|} \vec{r}_{s, \|} \Theta\left(k_{y}, p_{y}, \vec{r}_{s, \|}\right) d^{2} r_{s, \|} d^{2} p_{\|},}
\end{aligned}
$$

where

$$
\Theta\left(k_{y}, p_{y}, \vec{r}_{s, \|}\right)=\int_{-\infty}^{\infty} e^{i k_{y} y_{s}} \Psi_{p}\left[y_{s}, H\left(\vec{r}_{s, \|}\right)\right] \frac{m V_{r}\left(\vec{r}_{s}\right)}{2 \pi i \hbar^{2}} d y_{s} .
$$

The scattered-wave function in Eq. (29) must be integrated over all possible contributions [Eq. (1)] and inserted in the definition of the mutual coherence function [Eq. (2) of Ref. 4] to find the scattered mutual coherence function close to the sample surface. After neglecting the interference between 
the incident beam and the reflected/refracted beam and assuming the scattered mutual coherence function close to the sample surface is homogeneous, the sample structure factor can be found as a two-dimensional Fourier transform of the sample surface-correlation function

$$
S_{k}(\vec{p}, \vec{k})=\int e^{-i \vec{Q}_{\|} \cdot \vec{r}_{\|}} G_{s}\left(\vec{p}, \vec{k}, \vec{r}_{\|}\right) d^{2} r_{\|}
$$

defined by (details can be found in Ref. 16)

$$
G_{s}\left(\vec{p}, \vec{k}, \vec{r}_{\|}\right)=\int \Theta^{*}\left(k_{y}, p_{y}, \vec{r}_{s, \|}\right) \Theta\left(k_{y}, p_{y}, \vec{r}_{s, \|}+\vec{r}_{\|}\right) d^{2} r_{s, \|}
$$

Note that now the sample structure factor is a twodimensional Fourier transform of a sample surfacecorrelation function. Essentially this sample surfacecorrelation function is the one-dimensional Fourier transform ( $y$ direction) of the sample correlation function

$$
G_{s}\left(\vec{p}, \vec{k}, \vec{r}_{\|}\right)=\int e^{-i Q_{y} y} G_{r}\left(\vec{r}_{\|}+y \vec{e}_{y}\right) d y .
$$

The dependence of $R_{\text {out }}$ and $\Gamma_{\text {in }}$ on $y$ in Eq. (20) is the same as the factor $e^{-i Q_{y} y}$ so that the integral over $y$ reduces this equation to

$$
I_{d}=2 v_{p} \int_{y=0} G_{s}\left(\vec{p}, \vec{k}, \vec{r}_{\|}\right) R\left(\vec{r}_{1}, \vec{r}_{1}+\vec{r}_{\|}\right) d^{2} r
$$

where

$$
R\left(\vec{r}_{1}, \vec{r}_{1}+\vec{r}_{\|}\right)=R_{\text {out }}\left(\vec{r}_{1}, \vec{r}_{1}+\vec{r}_{\|}\right) \Gamma_{\text {in }}\left(\vec{r}_{1}, \vec{r}_{1}+\vec{r}_{\|}, 0\right) .
$$

Equations (31) and (34) hold for all Green's functions that can be cast in the form of Eq. (A17), not only the one derived here. In case of a substrate with a certain height profile (the layer is of the same material as the substrate), $\Psi_{p}\left[y_{s}, H\left(\vec{r}_{s, \|}\right)\right]$ reduces to the phase-object approximation

$$
\Psi_{p}\left(y_{s}, H\right)=\begin{array}{ll}
\tau^{a, s}\left(p_{y}\right) e^{i H\left(p_{y}^{s}-p_{y}\right)-i p_{y}^{s} y_{s}} & y_{s}<H \\
e^{-i p_{y} y_{s}}+e^{i p_{y}\left(y_{s}-2 H\right)} \rho^{a, s}\left(p_{y}\right) & y_{s} \geq H
\end{array}
$$

so that

$$
\Theta\left(k_{y}, p_{y}, \vec{r}_{s, \|}\right)=\frac{p_{y}}{2 \pi} \frac{p_{y}-p_{y}^{s}}{k_{y}-p_{y}^{s}} e^{i H\left(\vec{r}_{s, \|}\right)\left(k_{y}-p_{y}\right)} .
$$

For the First Born approximation one finds

$$
\Psi_{p}\left(y_{s}, H\right)=e^{-i p_{y} y_{s}}
$$

so that

$$
\Theta\left(k_{y}, p_{y}, \vec{r}_{s, \|}\right)=\frac{\rho_{b}}{k_{y}-p_{y}} e^{i H\left(\vec{r}_{s, \|}\right)\left(k_{y}-p_{y}\right)},
$$

where $\rho_{b}$ is the scattering length density $\left[4 \pi \rho_{b}=p_{y}^{2}-\left(p_{y}^{s}\right)^{2}\right]$. For the distorted-wave Born approximation as advocated by Sinha ${ }^{14}$

$$
\Psi_{p}\left(y_{s}, H\right)=\tau^{a, s}\left(k_{y}\right) \tau^{s, a}\left(p_{y}\right) e^{i\left(k_{y}^{s}-p_{y}^{s}-k_{y}\right) y_{s}}
$$

so that

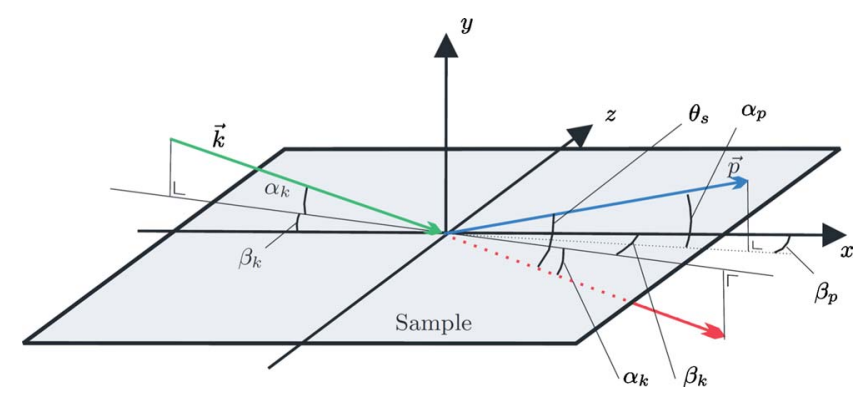

FIG. 4. (Color online) Definition of angles in spin-echo neutron reflection geometry. $\alpha_{k}$ is the angle between the path of the incident neutron (represented by the green arrow and vector $\vec{k}$, or the red arrow where the start of the vector has been shifted to the sample surface) and the sample surface. $\beta_{k}$ is the angle the path of the incident neutron makes with the $x y$ plane. $\alpha_{p}$ is the angle between the path of the off-specularly scattered neutron (blue arrow and vector $\vec{p}$ ) and the sample surface and $\beta_{p}$ is the angle between the path of the off-specularly scattered neutron and the $x y$ plane. $\theta_{s}$ is the angle between the path of the off-specularly scattered neutron and the path of the incident neutron.

$$
\Theta\left(k_{y}, p_{y}, \vec{r}_{s, \|}\right)=\tau^{a, s}\left(k_{y}\right) \tau^{s, a}\left(p_{y}\right) \frac{\rho_{b}}{k_{y}^{s}-p_{y}^{s}} e^{i H\left(\vec{r}_{s, \|}\right)\left(k_{y}^{s}-p_{y}^{s}\right)} .
$$

The count rate at the detector can be found by first determining the sample surface-correlation function by inserting this function in Eq. (32) and inserting it in Eq. (34) or by first calculating the Fourier transform to determine the sample structure factor in Eq. (31) and fold it with the instrumental resolution according to Eq. (22).

\section{SPIN-ECHO NEUTRON REFLECTOMETRY}

Rekveldt considered combining the neutron spin-echo technique and reflectometry. ${ }^{17}$ In the same way as in smallangle neutron scattering ${ }^{5}$ it is possible to use the precession of the neutron spin to code the angle of the neutron path through magnetic-flux-density regions. The influence of the magnetic-flux density is described by means of its influence on the precession along the classical neutron path through the instrument. ${ }^{4}$

To describe the neutron path in case of neutron reflectometry four angles are important: $\alpha_{k}$, the angle between the path of the incident neutron (wave vector $\vec{k}$ ) and the sample surface and the same for the not-scattered or transmitted neutron. $\beta_{k}$, the angle the path of the incident neutron makes with the $x y$ plane. $\alpha_{p}$ the angle between the path of the offspecularly scattered neutron (wave vector $\vec{p}$ ) and the sample surface and $\beta_{p}$ the angle between the path of the offspecularly scattered neutron and the $x y$ plane. These angles are schematically shown in Fig. 4. $\theta_{s}$ is the angle between the path of the off-specularly scattered neutron and the path of the not-scattered neutron. Using these definitions, the wave vector of the incident neutron is represented by

$$
\vec{k}=k\left(\begin{array}{c}
\cos \alpha_{k} \cos \beta_{k} \\
-\sin \alpha_{k} \\
\cos \alpha_{k} \sin \beta_{k}
\end{array}\right)
$$

and the wave vector of the scattered neutron by 


$$
\vec{p}=k\left(\begin{array}{c}
\cos \alpha_{p} \cos \beta_{p} \\
\sin \alpha_{p} \\
\cos \alpha_{p} \sin \beta_{p}
\end{array}\right) .
$$

The polarization precession angle acquired by a neutron traveling through parallelogram shaped magnetic-flux-density regions (with length, $L$ and strength, $B$ ) is given by

$$
\phi_{\mathrm{I}}\left(\vec{r}^{\prime}, \vec{r}\right)=c \lambda B L\left(1-\theta_{\mathrm{I}} \tan \theta_{0}\right)
$$

and

$$
\phi_{\mathrm{II}}\left(\vec{r}^{\prime}, \vec{r}\right)=-c \lambda B L\left(1-\theta_{\mathrm{II}} \tan \theta_{0}\right),
$$

where $c=4.63209 \times 10^{14} \mathrm{~T}^{-1} \mathrm{~m}^{-2}$ and $\theta_{0}$ is the angle between the $x$ axis and the faces of the magnets. The angle that is coded by the precession angle $\left(\theta_{\mathrm{I}}\right.$ or $\left.\theta_{\mathrm{II}}\right)$ is the angle the neutron path makes with the $x$ axis in the plane of the parallelogram (see also Fig. 6 of Ref. 4). The coded angle before the sample is $\beta_{k}$ and after the sample is either $\beta_{k}$ or $\beta_{p}$ depending on which neutron path is considered (the transmitted or the scattered one, respectively). Here, for a notscattered or specularly reflected neutron the precession angle acquired in region $\mathrm{I}$ is exactly balanced by the one in region II, producing a perfect spin echo. If $\beta_{p}$ is different from $\beta_{k}$ by scattering in the $z$ direction this exact balance is canceled and a net precession angle remains. This enables the probing of the sample surface structure in the $z$ direction. The inplane structure of the sample can also be determined by means of off-specular reflection as was discussed in the previous chapter. Then, the sample surface-structure factor is determined from the measurement of the neutron count rate at the relevant detector positions. As all direct scattering techniques this is done in momentum transfer-space or $Q$ space. Using the spin-echo technique this is converted to real space by an appropriate Fourier transform using the precession angle coding of the momentum transfer. This was first experimentally tested by Felcher et $a{ }^{18}$ in 2002 and repeated in 2003. ${ }^{19}$

As only elastic scattering is addressed here, the momentum transfer is completely determined by the scattering angle $\theta_{s}$, or the difference between $\beta_{k}$ and $\beta_{p}$. The propagation of the mutual coherence matrix is given by Eq. (27) of Ref. 4 where the matrices $\hat{D}$ are defined in Eq. (26) of Ref. 4 and describe the magnetic-flux-density interaction with the neutron-wave function traveling from $\overrightarrow{r_{1}^{\prime}}$ or $\overrightarrow{r_{2}^{\prime}}$ on the neutron source to $\vec{r}_{1}$ or $\vec{r}_{2}$ on the detector via the sample surface. As shown by de Haan ${ }^{4}$ the device matrix between polarizer and flipper for the spin-echo instrument here is given by

$$
\hat{E}\left(\vec{r}, \vec{r}^{\prime}\right)=\hat{R}\left(\vec{r}^{\prime}, \vec{r}\right)^{\dagger} \hat{T}_{z}\left[\phi_{\mathrm{II}}(\vec{p})\right] \hat{T}_{z}\left[\phi_{\mathrm{I}}(\vec{k})\right] \hat{R}\left(\vec{r}^{\prime}, \vec{r}\right),
$$

where $\hat{R}\left(\vec{r}^{\prime}, \vec{r}\right)$ is the device matrix of the rotator before region I and $\hat{R}\left(\vec{r}^{\prime}, \vec{r}\right)^{\dagger}$ the same for the rotator after region II and $\phi_{\mathrm{I}}$ and $\phi_{\mathrm{II}}$ are determined by Eqs. (43) and (44) taking $\theta_{\mathrm{I}}=\beta_{k}$ and $\theta_{\mathrm{II}}=\beta_{p}$. If the sample scatters nonmagnetic the device matrix for region I, the sample and region II is just $\hat{T}_{z}\left(\vec{l}_{s e} \cdot \vec{Q}\right)$, where as before $\vec{Q}=\vec{p}-\vec{k}$ is the wave-vector transfer at the sample position, the direction of $\vec{l}_{s e}$ is the coding direction (here $\vec{e}_{z}$ ) and $l_{s e}=c \lambda^{2} B L \tan \theta_{0} / 2 \pi$ is called the spin-echo length. The constant $c B L \tan \theta_{0} / 2 \pi$ is called the spin-echo length constant. The polarizing factor of $\hat{E}\left(\vec{r}, \vec{r}^{\prime}\right)$ is

$$
P_{E}\left(\vec{r}, \vec{r}^{\prime}\right)=\left[1-P_{R}^{2}\left(\vec{r}^{\prime}, \vec{r}\right)\right] \cos \left\{\vec{l}_{s e} \cdot \vec{Q}\right\}+P_{R}^{2}\left(\vec{r}^{\prime}, \vec{r}\right),
$$

where $P_{R}$ is the polarizing factor of $\hat{R}\left(\vec{r}^{\prime}, \vec{r}\right)$. For a perfect spin-echo instrument the factor before the cosine must be maximal and the other term minimal hence $P_{R}=0$. The complete device matrix is found by including the polarizer, flipper, and analyzer

$$
\hat{D}\left(\vec{r}, \vec{r}^{\prime}\right)=\hat{D}_{A}\left(\vec{r}, \vec{r}^{\prime}\right) \hat{F}\left(\vec{r}, \vec{r}^{\prime}\right) \hat{E}\left(\vec{r}, \vec{r}^{\prime}\right) \hat{D}_{P}\left(\vec{r}, \vec{r}^{\prime}\right),
$$

where $\hat{D}_{P}$ corresponds to the device matrix of the polarizer, $\hat{D}_{A}$ that of the analyzer, and $\hat{F}$ that of the flipper just before the analyzer. ${ }^{4}$ In the following it is assumed that these device matrices are constant. Hence $\hat{D}$ only depends on $\vec{p}-\vec{k}$. The detector count rate can be found by Eq. (8). The detector area is assumed to be perpendicular to $\vec{v}_{p}$ and assumed large enough to capture all scattered or reflected neutrons. This yields ${ }^{16}$ for a completely homogeneous, incoherent, and unpolarized source with area $A_{0}$

$$
I_{d}=\int_{A_{d}} \int_{A_{0}} \frac{J_{0} \Omega_{d}(\vec{p}-\vec{k})}{4 \pi r_{d s}^{2} r_{s 0}^{2}} S_{k}(\vec{p}, \vec{k}) d^{2} r^{\prime} d^{2} r_{d}
$$

where

$$
\Omega_{d}(\vec{p}-\vec{k})=\frac{1}{2} \operatorname{Tr}\left[\hat{D}(\vec{p}-\vec{k}) \hat{D}(\vec{p}-\vec{k})^{\dagger}\right]
$$

and $\vec{k}=k\left(\vec{r}_{s}-\vec{r}^{\prime}\right) / r_{s}, \vec{p}=k\left(\vec{r}_{d}-\vec{r}_{s}\right) / r_{d s}$, and $\vec{r}^{\prime}$ is the source position. It was assumed that the sample dimensions were small compared to the distance between sample and source and between detector and sample. The two-shim count rate can be determined by replacing $\Omega_{d}$ in the above equation by $T_{P} T_{A} / 4$ where $T_{P}$ and $T_{A}$ are the transmissions of polarizer and analyzer, respectively,

$$
I_{s}=\frac{T_{P} T_{A}}{4} \int_{A_{d}} \int_{A_{0}} \frac{J_{0}}{4 \pi r_{d s}^{2} r_{s}^{2}} S_{k}(\vec{p}, \vec{k}) d^{2} r^{\prime} d^{2} r_{d} .
$$

The two-flip count rate is found by replacing $\Omega_{d}$ by $T_{P} T_{A} P_{P} P_{A} P_{E}(\vec{p}-\vec{k}) / 4$. The integral over the detector area can be transformed to an integral over $p_{y}$ and $p_{z}$ and the integral over the source area can be transformed to an integral over $k_{y}$ and $k_{z}$

$$
\begin{aligned}
I_{f}= & \frac{T_{P} T_{A} P_{P} P_{A}}{4} \frac{J_{0}}{4 \pi k^{4}} \\
& \times \iiint \int S_{k}(\vec{p}, \vec{k}) P_{E}(\vec{p}-\vec{k}) d p_{z} d p_{y} d k_{z} d k_{y} .
\end{aligned}
$$

This integral can be evaluated by realizing that in the case considered $P_{E}$ only depends on the difference $p_{z}-k_{z}$. Further, the sample structure factor only depends on $\vec{p}-\vec{k}$ and $k_{y}$ and $p_{y}$. If the detector is wide enough the integral over $p_{z}$ goes from $-\infty$ to $+\infty$ and can be replaced by an integral over $Q_{z}$ $=p_{z}-k_{z}$ and the same range 


$$
I_{f}=\frac{T_{P} T_{A} P_{P} P_{A}}{4} \frac{J_{0}}{4 \pi k^{2}} \int d k_{z} \int \widetilde{S}_{k}(\vec{p}, \vec{k}) P_{E}\left(Q_{z}\right) d Q_{z},
$$

where

$$
\tilde{S}_{k}(\vec{p}, \vec{k})=\frac{1}{k^{2}} \iint S_{k}(\vec{p}, \vec{k}) d k_{y} d p_{y}
$$

and the measured polarization becomes

$$
P_{m}=P_{P} P_{A} \frac{\int \tilde{S}_{k}(\vec{p}, \vec{k}) P_{E}\left(Q_{z}\right) d Q_{z}}{\int \tilde{S}_{k}(\vec{p}, \vec{k}) d Q_{z}} .
$$

The factor $P_{E}\left(Q_{z}\right)$ is given by Eq. (46). In the ideal spin-echo instrument $P_{R}=0$ and the integral over $Q_{z}$ represents a cosine transform (or the real part of a Fourier transform) of the sample structure factor hence the above equation becomes

$$
P_{m}=P_{P} P_{A} \frac{G\left(l_{s e}\right)}{G(0)},
$$

where

$$
G\left(l_{s e}\right)=k^{2} \Re\left[\int \tilde{S}_{k}(\vec{p}, \vec{k}) e^{i l_{s e} Q_{z}} d Q_{z}\right]
$$

comparable to the result obtained by spin-echo small-angle neutron scattering. ${ }^{4} G(r)$ is a one-dimensional sample correlation function. For standard neutron-reflection geometry (see Fig. 1) the range of integration of $k_{y}$ is determined by position $H$ and the width $W_{y}$ of the entrance diaphragm and the range of integration of $p_{y}$ by position $H_{d}$ and the width $D_{y}$ of the diaphragm before the detector. In general these ranges are quite small and the integration over $k_{y}$ and $p_{y}$ can be interpreted as a resolution effect. Ignoring these resolution effects one finds

$$
\frac{P_{m}}{P_{P} P_{A}}=\frac{\int e^{-i Q_{x}^{x}} G_{s}\left(\vec{p}, \vec{k}, l_{s e} \vec{e}_{z}+x \vec{e}_{x}\right) d x}{\int e^{-i Q_{x} x} G_{s}\left(\vec{p}, \vec{k}, x \vec{e}_{x}\right) d x},
$$

which reduces for a one-dimension grating perfectly aligned with the beam direction to

$$
\frac{P_{m}}{P_{P} P_{A}}=\frac{G_{s}\left(\vec{p}, \vec{k}, l_{s e}\right)}{G_{s}(\vec{p}, \vec{k}, 0)} .
$$

One should realize that the validity of Eq. (56) depends on several conditions, which were mentioned during its derivation. The most important ones are the quality of the spinecho instrument, which determines $P_{E}\left(Q_{z}\right)$ and the small size of the sample compared to the distance between sample and source and between sample and detector. In (spin-echo) small-angle neutrons-scattering instruments this condition is almost always met. For neutron reflectometers large sample sizes are more common. In case were the sample size is too large deviations might occur and one should use Eq. (48) instead. Another important condition is that the sample cor-

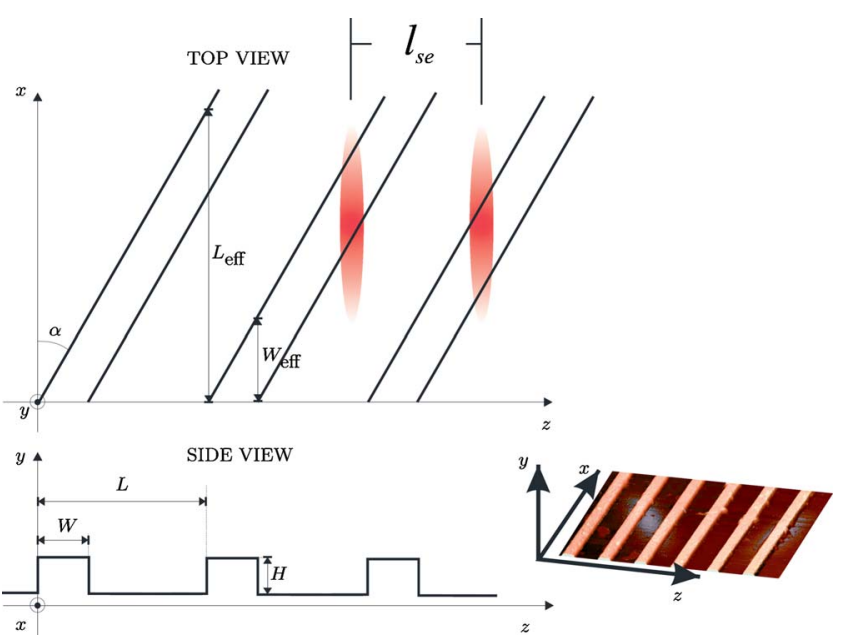

FIG. 5. (Color online) Top view and side view of the samples. $x$ is the beam direction, $y$ is perpendicular to the sample surface, and $z$ is perpendicular to the beam direction and parallel to the sample plane. They are asymmetric silicon block profiles on a silicon substrate with period of $L=900 \mathrm{~nm}$ and height, $H=80 \mathrm{~nm}$ (sample A) and $40 \mathrm{~nm}$ (sample B), respectively. The width, $W$ of the block compared to the total period is for both samples 2:9. The in-plane angle between beam direction and ridges is $\alpha$, resulting in an effective period $L_{\text {eff }}=L / \sin \alpha$ and width $W_{\text {eff }}=W / \sin \alpha$. The sample areas were approximately $2 \mathrm{~cm}^{2}$. The red ellipses show the coherence areas of the spin-up and spin-down wave functions.

relation length that is probed $\left(l_{s e}\right)$ is smaller than $\sqrt{r_{d s} / k}$. Otherwise the equations used to calculate the scattered coherence function should be calculated using its definition and the scattered-wave functions. Finally, the magnetic-flux density should be homogeneous over the probed length, otherwise the way the coherence matrix propagates is not accurately described by Eq. (30) of Ref. 4. In that case no analytical solution has been found for the propagation of the mutual coherence matrix or (which is the same) polarization of the neutron beam.

\section{EXPERIMENTS}

Several experiments were performed on two samples. The sample shape is shown in Fig. 5. The shape was designed as an asymmetric silicon block profile on a silicon substrate with period, $L$ of $900 \mathrm{~nm}$ and height, $H$ of 80 (sample A) and $40 \mathrm{~nm}$ (sample B), respectively. The width, $W$ of the block compared to the total period was designed for both samples 2:9 hence $W$ equals $200 \mathrm{~nm}$. The sample areas were approximately $2 \mathrm{~cm}^{2}$. Atomic force microscopy (AFM) measurements were done to check the design parameters at the real samples. The results are shown in Table II. An example is shown as an inset in Fig. 5. The period and width of the sample are close to their design values but the heights of the samples deviate considerably. This is probably due to the etching process used to manufacture the sample. The sample surface-correlation function for these block-shaped samples according to the phase-object approximation determined by inserting Eq. (36) in Eq. (32) is given by 
TABLE II. Neutron-reflectometry results, measurements details, and sample parameters (coherence length for $\lambda=0.15 \mathrm{~nm})$.

\begin{tabular}{lccccc}
\hline \hline Measurement & 87 & 88 & 95 & 96 & 98 \\
\hline Sample & $\mathrm{A}$ & $\mathrm{A}$ & $\mathrm{A}$ & $\mathrm{A}$ & $\mathrm{B}$ \\
$\theta$, incident angle/mrad & 3.53 & 6.98 & 3.5 & 3.59 & 3.48 \\
$\Delta \theta$, resolution incident angle/mrad & 0.07 & 0.26 & 0.10 & 0.13 & 0.10 \\
$\alpha$, in-plane angle & $6.7^{\circ}$ & $6.7^{\circ}$ & $3.9^{\circ}$ & $2.1^{\circ}$ & $2.7^{\circ}$ \\
$H$, designed height/nm & 80 & 80 & 80 & 80 & 40 \\
AFM height & $100(2)$ & $100(2)$ & $100(2)$ & $100(2)$ & $61(3)$ \\
Fitted height & $98(2)$ & $98(2)$ & $99(2)$ & $99(2)$ & $56(2)$ \\
$L$, period/ $\mu \mathrm{m}$ & 0.90 & 0.90 & 0.90 & 0.90 & 0.90 \\
$W$, designed width/ $\mu \mathrm{m}$ & 0.20 & 0.20 & 0.20 & 0.20 & 0.20 \\
AFM width & $0.30(5)$ & $0.30(5)$ & $0.30(5)$ & $0.30(5)$ & $0.30(5)$ \\
Fitted width & $0.21(5)$ & $0.23(7)$ & $0.23(5)$ & $0.21(5)$ & $0.23(9)$ \\
Coherence length/ $\mu \mathrm{m}$ & 100 & 14 & 70 & 50 & 70 \\
\hline \hline
\end{tabular}

$$
G_{s}\left(\vec{r}_{\|}\right)=\frac{p_{y}^{2} A_{s}}{4 \pi^{2}} R_{P O}(\vec{p}, \vec{k}) g_{s}\left(\vec{r}_{\|}\right),
$$

where $R_{P O}(\vec{p}, \vec{k})=\left|\left(p_{y}-p_{y}^{s}\right) /\left(k_{y}-p_{y}^{s}\right)\right|^{2}$ and $g_{s}\left(\vec{r}_{\|}\right)$is given by

$$
\begin{array}{ll}
1-2 \frac{\vec{r}_{\perp} \cdot \vec{n}_{\|}}{L}\left[1-\cos \left(Q_{y} H\right)\right] & 0<\vec{r}_{\|} \cdot \vec{n}_{\perp}+m L<W \\
1-\frac{2 W}{L}\left[1-\cos \left(Q_{y} H\right)\right] & \text { otherwise }
\end{array}
$$

with $Q_{y}=p_{y}-k_{y}, \vec{n}_{\perp}$ the unit vector perpendicular to the direction of the grating and $m$ the largest integer smaller than $\vec{r}_{\|} \cdot \vec{n}_{\perp} / L$. The corresponding sample structure factor can be split in a specular and diffuse part

$$
S_{k}(\vec{p}, \vec{k})=S^{S}(\vec{p}, \vec{k})+S^{D}(\vec{p}, \vec{k})
$$

given by

$$
\begin{aligned}
S^{S}(\vec{p}, \vec{k})= & p_{y}^{2} A_{s} R_{P O}(\vec{p}, \vec{k}) \delta^{2}\left(\vec{Q}_{\|}\right) \\
& \times\left\{1-\frac{2 W}{L}\left(1-\frac{W}{L}\right)\left[1-\cos \left(Q_{y} H\right)\right]\right\}
\end{aligned}
$$

and

$$
\begin{aligned}
S^{D}(\vec{p}, \vec{k})= & p_{y}^{2} A_{s} R_{P O}(\vec{p}, \vec{k}) \delta\left(\vec{Q}_{\|} \cdot \vec{n}_{\|}\right) 2\left[1-\cos \left(Q_{y} H\right)\right] \\
& \times \sum_{m=-\infty, m \neq 0}^{m=\infty} \delta\left(\vec{Q}_{\|} \cdot \vec{n}_{\perp}-\frac{2 \pi m}{L}\right) \frac{\sin ^{2}\left(\frac{m \pi W}{L}\right)}{m^{2} \pi^{2}},
\end{aligned}
$$

where $\vec{n}_{\|}$is the unit vector parallel to the direction of the grating. Note that for the specular part $R_{P O}(\vec{p}, \vec{k})$ reduces to the Fresnel reflectivity of the air-substrate interface.

\section{A. Neutron reflectivity}

Neutron-reflectivity measurements were performed on reflectometer CRISP of ISIS/RAL..$^{20}$ Measurements were done on sample $\mathrm{A}$ at an incident angle of $3.5 \mathrm{mrad}$ and three angles of the ridges compared to the incident beam (this in-plane angle between the incident-beam angle and the ridges, $\alpha$ was $6.7^{\circ}, 3.5^{\circ}$ and $2.3^{\circ}$, respectively). The details of the measurements are shown in Table II. The measurements and theoretical calculations according to the phaseobject approximation are shown in Fig. 6. A further measurement was done on this sample at an incident angle of 7.0 mrad and an in-plane angle of $6.7^{\circ}$. The measurement and theoretical calculations are shown in Fig. 7.

The theoretical calculations were done according to Eq (22) by inserting Eq. (60) and using a simplified Gaussian instrumental resolution function. In contrast to Eq. (4) the instrumental resolution function is taken as the product of two orthogonal resolution functions to simplify the calculations. A Gaussian resolution function in $Q_{x}$ was used. The standard deviation of the distribution was given by the spread in incident angles, $\Delta \theta$ (given in Table II) so that $\sigma_{Q_{x}}=2 \pi \theta \Delta \theta / \lambda$. Hence, the corresponding mutual coherence function was represented by a Gaussian distribution with a standard deviation (or effective coherence length) $r_{x}$ $=\left(\sigma_{Q_{x}}\right)^{-1}$. The coherence length in the direction perpendicular to the $x$ direction in the sample plane was taken very small and the corresponding instrumental resolution function represented by a $\delta\left(Q_{z}\right)$ function.

Measurements of both the specular and the off-specular intensity show qualitatively the same features as calculations according to the phase-object approximation. As is mentioned in the Appendix the calculation of the Green's function is strictly speaking only valid under conditions that are not always satisfied, especially for the specular part. The specular part $S^{S}$ of the scattering can be substituted by the specular reflectivity calculated by assuming a layer of the same height as the samples ridges and an average scattering potential so that

$$
S^{S}(\vec{p}, \vec{k})=p_{y}^{2} A_{s}\left|\frac{r_{0}^{F}+r_{1}^{F} e^{2 i k_{l} H}}{1+r_{0}^{F} r_{1}^{F} e^{2 i k_{l} H}}\right|^{2} \delta^{2}\left(\vec{Q}_{\|}\right),
$$

where $k_{l}$ is the perpendicular component of the wave vector inside the layer, $r_{0}^{F}$ is the Fresnel reflection coefficient from 

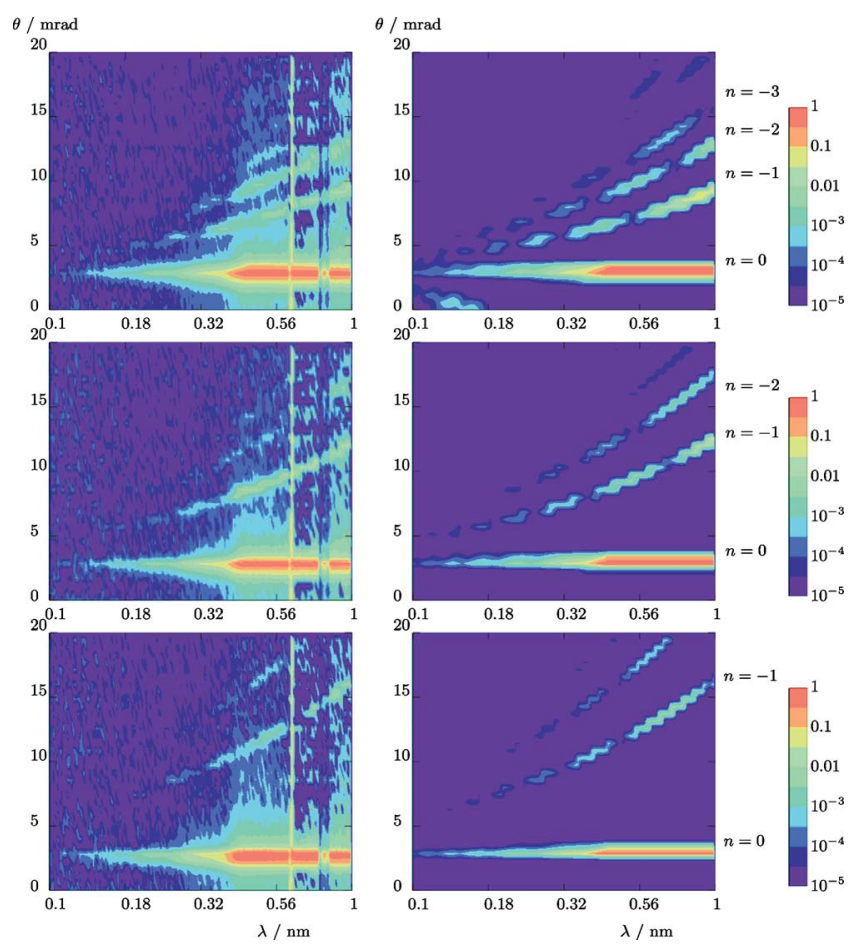

FIG. 6. (Color online) Normalized neutron count rate at detector as measured (left) and calculated (right) for sample A and incident angle of $3.5 \mathrm{mrad}$ and in-plane angle $6.7^{\circ}, 3.5^{\circ}$, and $2.3^{\circ}$ as function of scattering angle $\theta$ and wavelength (the wavelength scale was renormalized to constant $\Delta \lambda / \lambda$ to increase the statistics (note the logarithmic wavelength scale). Note the intensity is located at $Q_{x}$ $=2 \pi n / L_{\text {eff }}$ for orders $n=0,-1,-2$, and -3 . The angle-independent structure between $\lambda=0.6$ and $0.8 \mathrm{~nm}$ is due to increased background of the (insufficiently blocked) second pulse from the ISIS target.

air to layer and $r_{1}^{F}$ the reflection coefficient from layer to substrate. For the above measurements this gives a more accurate description of the specular reflectivity. The fitted parameters are shown in Table II. The fitted heights agree within the errors to the heights determined with AFM measurements. The fitted widths are smaller than the AFM measurements but within error bars equal to the design values. Probably the AFM width is overestimated due to the size of the AFM tip $(\approx 10 \mathrm{~nm})$. The phase-object approximation de-
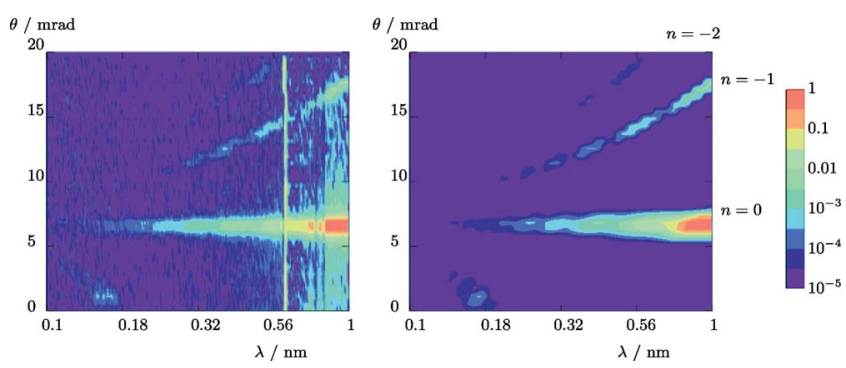

FIG. 7. (Color online) Normalized neutron count rate at detector as measured (left) and calculated (right) for sample A and incident angle of $7.0 \mathrm{mrad}$ and in-plane angle $6.7^{\circ}$ as function of scattering angle $\theta$ and wavelength $\lambda$. Note the intensity is located at $Q_{x}$ $=2 \pi n / L_{\text {eff }}$ for orders $n=0,-1$, and -2 . The same comments hold as in the caption of Fig. 6.

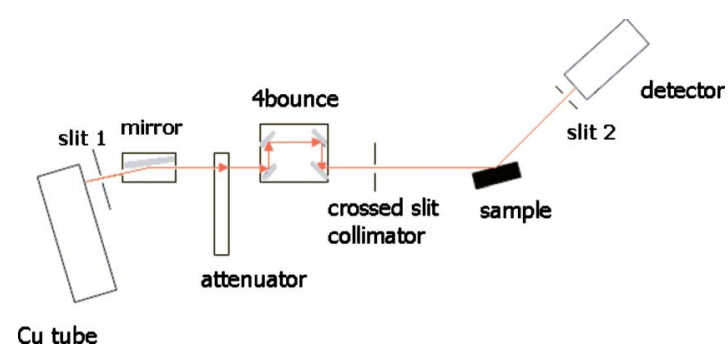

FIG. 8. (Color online) X-ray measurement setup. From left to right: the X-ray tube $\mathrm{Cu}$ with line focus (PW3373/00) used as the source, slit 1 (width $0.5^{\circ}$ ), x-ray mirror (PW3088/60) with an equatorial divergence $<0.05^{\circ}$, an automatic beam attenuator (PW 3087/ $60)$, an asymmetrical four-crystal Ge 220 monochromator (PW3098/27) used to obtain $\mathrm{Cu} K \alpha_{1}$ radiation $(\Delta \lambda / \lambda=23 \mathrm{ppm})$ with beam divergence of $0.005^{\circ}$, a crossed slit collimator (PW3143/ 20) with axial height of $5 \mathrm{~mm}$ and equatorial width of $1 \mathrm{~mm}$, the goniometer with a radius of $360 \mathrm{~mm}$ (PW3050/65), slit 2 $\left(0.016^{\circ} 2 \theta\right)$, and a proportional gas-filled detector (PW3011/20).

viates close to the critical edge. The calculations in this region are very sensitive to the exact resolution function (or mutual coherence function) at the sample position and of the sample surface-correlation function.

\section{B. X-ray reflectivity}

$\mathrm{X}$-ray reflectivity measurements were performed with a Panalytical Xpert Pro MRD X-Ray diffractometer at Material Science and Engineering Department of the Delft University of Technology. The used setup is shown in Fig. 8. See its caption for component details. Several measurements were done with different in-plane angles. Figure 9 shows the measurement and theoretical calculations for sample A. The angle between the incident beam and the profile was approximately $10^{\circ}$. The scattered intensity was calculated in a similar way as described for the neutron-reflectivity measurements, using the parameters shown in Tables II and III. Figure 10 shows a similar measurement and theoretical calculation on sample B. The relevant features of the measurements are clearly reproduced by the phase-object approximation. The different positive and negative orders are at the same position and their intensities relative to each other are calculated quite accurately. The intensity oscillations along
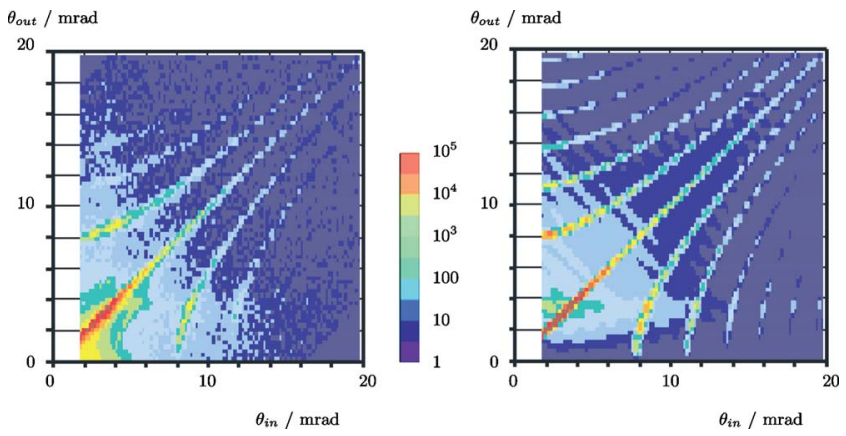

FIG. 9. (Color online) Measured (left) and calculated (right) $\mathrm{x}$-ray intensity at detector for sample A. Note the color scale is logarithmic. 
TABLE III. X-ray measurement details (coherence length for $\lambda=0.15 \mathrm{~nm})$.

\begin{tabular}{lcc}
\hline \hline Parameter & Sample A & Sample B \\
\hline In-plane angle/degrees & 10.5 & 9.7 \\
Angular resolution/mrad & 0.077 & 0.077 \\
Coherence length/ $\mu \mathrm{m}$ & 91 & 91 \\
Isotropic roughness/nm & 0.6 & 1.1 \\
\hline \hline
\end{tabular}

the orders due to the sample height and optical phase matching of the reflection at the top and bottom of the profile, have the same position and frequency. Some deviations occur. Especially close to and below the critical edge and in the regions between the orders, and the relative intensity of the orders with respect to the specular order. This could be due to several factors such as the sample surface roughness, aperiodicity of the grating or inaccurate estimate of the incidentbeam-resolution function. Further due to the limited size of the diaphragm before the detector it is possible that some $\mathrm{x}$-rays are scattered out of the beam and not detected. In the calculations a self-affine roughness ${ }^{21}$ was added using a roughness standard deviation of $1.1 \mathrm{~nm}$ for sample A and 0.6 $\mathrm{nm}$ for sample B with a correlation length of $1000 \mathrm{~nm}$ and a jaggedness of 1 . Although this roughness clearly shows up as off-specular intensity between the orders it only roughly corresponds to the measured values indicating that the actual sample roughness deviates from the simple model considered here. This also obstructs further detailed comparison between measurements and calculations.

\section{Neutron spin-echo reflectivity}

Neutron spin-echo measurements were performed with sample B on reflectometer OffSpec of ISIS/RAL. ${ }^{22-24}$ As this is a time-off-flight reflectometer, the spin-echo length is proportional to the square of the wavelength. For the measurements here the spin-echo length constant was set at $3.05 \mu \mathrm{m} / \mathrm{nm}^{2}$. Again measurements for several in-plane angles were done for a fixed incident angle, $\theta=3.4 \mathrm{mrad}$. The normalized shim count rate for $\alpha=0^{\circ}$ and $\alpha=0.8^{\circ}$ are shown in Fig. 11. For $\alpha=0.8^{\circ}$ the specular reflectivity and also two negative orders and one positive order can be seen.
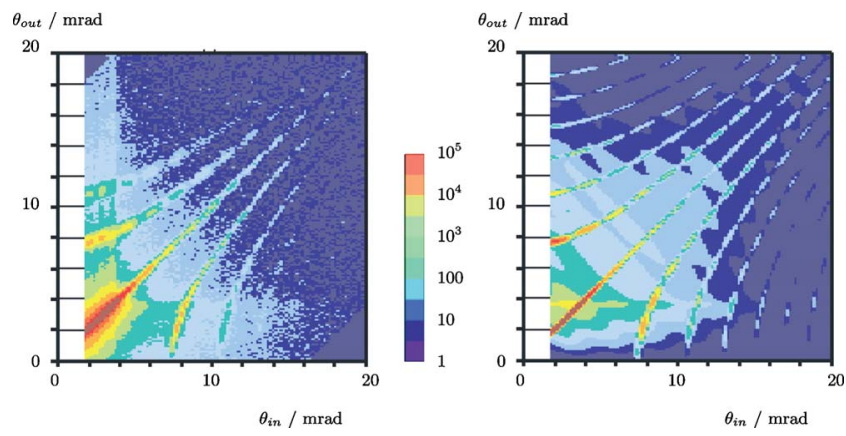

FIG. 10. (Color online) Measured (left) and calculated (right) $\mathrm{X}$-ray intensity at detector for sample B. Note the color scale is logarithmic.
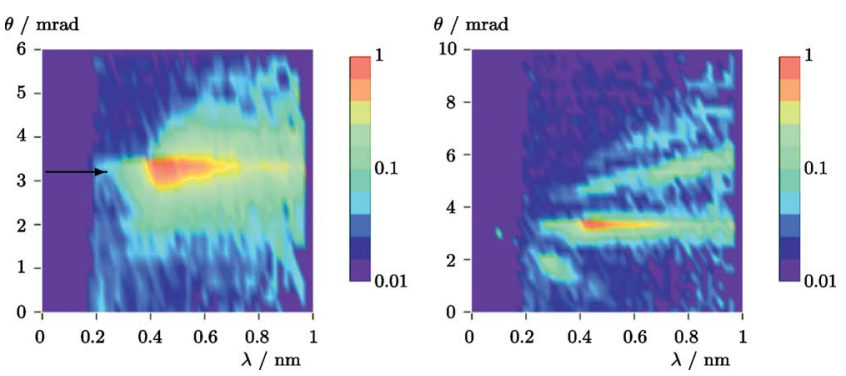

FIG. 11. (Color online) Measured normalized shim count rate for sample B and incident angle of $3.4 \mathrm{mrad}$ and in-plane angles $0^{\circ}$ (left) and $0.8^{\circ}$ (right) (estimated in-plane angle divergence $0.15^{\circ}$ ) as function of scattering angle $\theta$ and wavelength $\lambda$. The arrow shows the position of the cut of Fig. 14.

When $\alpha$ is reduced to 0 , the orders merge toward the same position as the specular reflectivity. The increased width of the specular reflectivity is due to the divergence in $\alpha$.

The normalized measured polarization is also measured and shown in Fig. 12. For $\alpha=0.8^{\circ}$ the polarization of the specular part is 1 . This is due to the fact that for the truly specular part $Q_{z}=0$ and hence $\beta_{p}=\beta_{k}$ so that the rotation built up in the first arm of the spin-echo spectrometer is completely reversed in the second arm. The polarization along the orders oscillates between +1 and -1 as shown for the -1 order in Fig. 13. This can be understood by looking at the diffuse part of the surface-scattering function given by Eq. (60). Inserting this function in Eq. (54) yields

$$
\frac{P_{m}}{P_{P} P_{A}}=\cos \left(2 \pi m \frac{l_{s e} \cos \alpha}{L}\right)
$$

under the condition that $Q_{x}=2 \pi m \sin \alpha / L$, which is fulfilled for the $m$ th order. Hence, the polarization along order $m$ oscillates as a cosine function of the spin-echo length with a period of $L / m($ as $\cos \alpha \approx 1)$ and an amplitude of 1 . This also explains the large oscillations in the polarization in the region just below or above the specular ridge for the measurements at $\alpha=0$. Note that for a one-dimensional height profile the shape and period of these oscillations are completely independent from the precise form of the sample structure factor (and hence also from the scattering theory used). It is
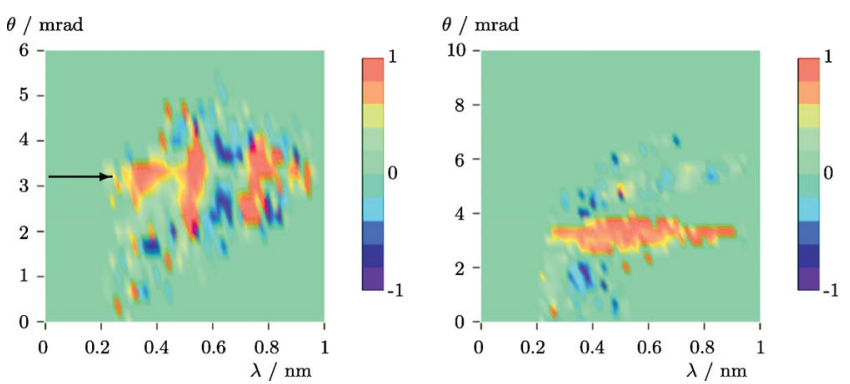

FIG. 12. (Color online) Measured normalized polarization for sample B under the same conditions as Fig. 11. Everywhere in the plot where the error bars on the polarization were larger than the polarization, the polarization was changed to 0 for display purposes. The black arrow shows the position of the cut of Fig. 14. 


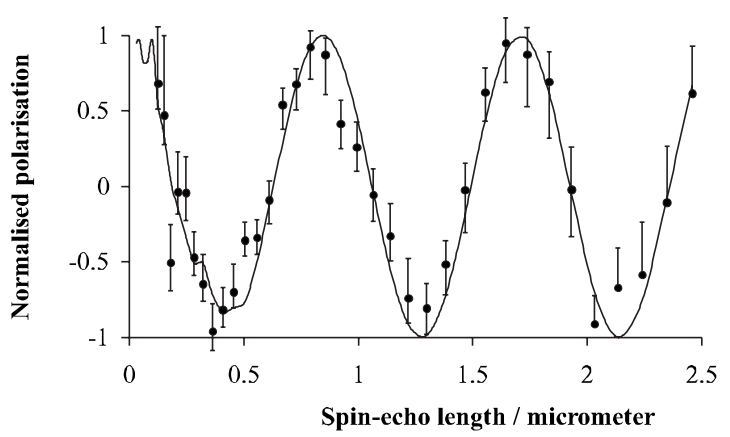

FIG. 13. Measured (dots and error bars) and calculated (line) normalized polarization along the order -1 (indicated in Fig. 12 as white arrow) as function of spin-echo length for sample B for an in-plane angle of $0.8^{\circ}$.

only needed that the different orders do not overlap. Hence, these gratings are extremely suitable for calibrating the spinecho length constant of these kinds of spectrometers.

For the measurement at $\alpha=0^{\circ}$ the specular cut of the normalized polarization as function of spin-echo length is shown in Fig. 14. The black continuous line is a model calculation using the phase-object approximation for the offspecular and specular scattering, determined by inserting Eq. (59) in Eq. (58). The fitted values for the model were $H$ $=50(1) \mathrm{nm}$ and $W=0.27(2) \mu \mathrm{m}$. Although the fit is good, the fitted parameters deviate considerably from the corresponding AFM values. This shows again that conditions under which the phase-object approximation holds are not completely satisfied. The (red) dashed line is a model calculation using the phase-object approximation for the off-specular and the reflectivity of the average scattering-length-density profile for the specular scattering as was done for the calculations of the normal off-specular measurements as shown before in Sec. V A. This can be done by inserting Eq. (60) in Eq. (54) and using Eq. (53). Interestingly, now the phaseobject approximation for the specular reflectivity is more accurate than the values from the average scattering-lengthdensity profile. The reason for this must be sought in the fact

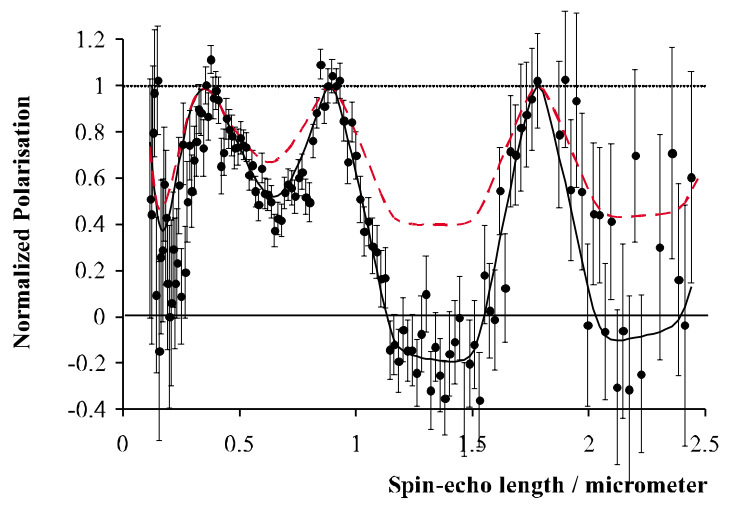

FIG. 14. (Color online) Measured (dots and error bars) and calculated (lines) normalized polarization as function of spin-echo length for sample B. Black line: completely according to phaseobject approximation, red dashed line: specular reflectivity calculated from average scattering-length-density profile. Grating is perfectly aligned with incident beam. that the distance between the ridges becomes larger (toward infinity due to $\alpha \rightarrow 0$ ) and the averaging distance for the scattering-length-density profile is smaller than this. One should realize that this averaging distance is not related to the coherence length of the neutron beam but to the extinction length of the neutron in the sample material. ${ }^{25}$ Only the sample volume within the extinction length contributes considerably to the reflected beam. The extinction length is on the order of $\left(\lambda \rho_{b}\right)^{-1}$, where $\rho_{b}$ is the scattering-length density of the sample material. For silicon and a wavelength of 0.2 $\mathrm{nm}$, the extinction length is some $20 \mu \mathrm{m}$, hence one should average the scattering-length-density profile over this length scale. As the grating period is $900 \mathrm{~nm}$, the region for the transition angle is at $\arcsin (0.9 / 20)=2.6^{\circ}$ for $0.2 \mathrm{~nm}$ neutrons and increasing linearly with wavelength. This explains also why, even when the conditions of the Appendix are not completely satisfied, the phase-object approximation produces correct results for the normalized polarization when the structures in the direction of the beam are longer than this extinction length.

The instrumental resolution has been applied in the same way as discussed for the previous experiments. The fact that one can still measure the sample correlations over distances much larger than the coherence length of the beam is due to the splitting of the wave function in a spin-up and spin-down wave function. The splitting is accomplished by the first spin-echo arm of the spectrometer. The distance on the sample between the coherence areas of the spin-up and the spin-down wave functions is equal to the spin-echo length, $l_{s e}$ and is much larger than the coherence length of the neutron beam in that direction. This is shown schematically as the red shaded areas in Fig. 5. Both wave functions are still able to interfere at the detector position as the splitting is reversed in the second spin-echo arm of the spectrometer, resulting in overlapping coherence areas.

\section{CONCLUSIONS}

It is possible to use the coherence theory to describe the propagation of neutrons through neutron or x-ray reflectometers. The coherence theory describes the propagation of the ensemble average of the neutron-wave function. The coherence theory as adopted here, only considers neutron-wave functions, having approximately an equal amount of total energy, denoted by (quasi-) monochromatic wave functions. This is due to the dispersion relation of matter waves and hence the interference between nonmonochromatic wave functions can, in general, be ignored. X-rays in vacuum have no dispersion, however the same limitation holds as the $\mathrm{x}$-rays are dispersive in a medium with finite-scattering potential. For reflectometry the coherence theory makes clear which approximations are used to obtain the standard results. It has been made clear which conditions must be fulfilled to be able to interpret the detector intensity as a folding of the sample correlation function with the instrumental resolution function. Especially the homogeneous coherence function at the sample position and the limited size of the sample surface-correlation function ensure that these conditions are met. 
Further it was shown that neutron-polarization effects can be described by using the coherence matrix approach. The interpretation of the neutron spin-echo reflectivity signal as a one-dimensional sample correlation function has been derived, again with the conditions needed for this interpretation.

The phase-object approximation has been extended for neutron and x-rays reflectivity geometry and compared with measurements of neutron reflectivity, x-ray reflectivity, and neutron spin-echo reflectivity on silicon gratings. It has been found that in the description of neutron spin-echo reflectivity measurements, the extinction length of the neutrons inside the sample material might play an important role.

\section{ACKNOWLEDGMENTS}

This research project has been supported in part by the European Commission under the 6th Framework Program through the Key Action: Strengthening the European Research Area, Research Infrastructures (Contract No. RII3CT-2003-505925) and by the Austrian Science Fund (Project No. F 1514). This work was financially supported by the Nederlandse Organisatie voor Wetenschappelijk Onderzoek (NWO).

\section{APPENDIX: SINGLE-LAYER GREEN'S FUNCTION}

In the most general case for elastic scattering, propagation of the wave function itself in the sample is governed by the stationary Lippmann-Swinger equation. ${ }^{9}$ The basis of the Lippmann-Schwinger equation is the time-dependent Schrödinger equation applied to a time-dependent potential. The potential can be factorized in a time-dependent part and a space-dependent part: $V(\vec{r}, t)=V_{r}(\vec{r}) V_{t}(t)$. By varying $V_{t}(t)$ in a specific way ( 0 for $t \rightarrow \pm \infty$ and 1 during the scattering process), the channel state can be identified as the solution of the Schrödinger equation before the scattering process and the scattered state as the solution after the scattering process. ${ }^{12}$ The Lippmann-Schwinger equation can also be derived from the stationary Schrödinger equation assuming appropriate boundary conditions (an incident plane wave and a scattered spherical wave at the sample position). The result is 9

$$
\Psi_{s c}(\vec{r})=\Psi_{i n}(\vec{r})-\frac{2 m}{\hbar^{2}} \int G_{0}\left(\vec{r}-\vec{r}_{s}\right) V_{r}\left(\vec{r}_{s}\right) \Psi_{s c}\left(\vec{r}_{s}\right) d^{3} r_{s},
$$

where $\Psi_{i n}(\vec{r})$ represents the channel state or incident wave function, $\Psi_{s c}(\vec{r})$ the scattered state or wave function, $V_{r}\left(\vec{r}_{s}\right)$ the scattering potential of the sample and $G_{0}(\vec{r})$ is the freeparticle Green's function ${ }^{26}$ given by

$$
G_{0}(\vec{r})=\frac{e^{i k r}}{4 \pi r} .
$$

In general the scattered-wave function is unknown. However, the Lippmann-Schwinger equation can be iterated resulting in an infinite series for $\Psi_{s c}(\vec{r})$ which can be rewritten in the form

$$
\Psi_{s c}(\vec{r})=\Psi_{i n}(\vec{r})-\frac{2 m}{\hbar^{2}} \int G^{(+)}\left(\vec{r}, \vec{r}_{s}\right) V_{r}\left(\vec{r}_{s}\right) \Psi_{i n}\left(\vec{r}_{s}\right) d^{3} r_{s},
$$

where $G^{(+)}\left(\vec{r}, \vec{r}_{s}\right)$ is a specialized (potential dependent) Green's function describing the scattering into a scatteredwave function and defined by the solution of the following Schrödinger equation

$$
\left[\nabla^{2}+k^{2}-\frac{2 m V_{r}\left(\vec{r}_{s}\right)}{\hbar^{2}}\right] G^{(+)}\left(\vec{r}, \vec{r}_{s}\right)=\delta\left(\vec{r}-\vec{r}_{s}\right),
$$

where the $\nabla^{2}$ operator represents the derivatives to $\vec{r}_{s}$. This equation describes the scattered wave as a superposition of waves produced by many scattering events occurring at different elements of the sample. This Green's function can be approximated by taking the Green's function for the undisturbed potential at position $(x, z)$ along the sample surface. For a layer with a variable height, $H$ on top of a substrate of a different material the Green's function is derived below. To find the solution a precise estimate of the Green's function and the undisturbed scattered-wave function is needed. Here the Green's function for a single layer on a substrate is derived and implemented to find the scattered wave. The Green's function for scattered waves can be approximated by taking the Green's function for scattered waves for the undisturbed potential ${ }^{13,27,28}$ defined by

$$
\left[\nabla^{2}+k^{2}-\frac{2 m V^{(0)}\left(\vec{r}_{s}\right)}{\hbar^{2}}\right] G^{(+)}\left(\vec{r}, \vec{r}_{s}\right)=\delta\left(\vec{r}-\vec{r}_{s}\right),
$$

where the $\nabla^{2}$ operator represents the derivatives to $\vec{r}_{s}$. It can be solved by assuming that the Green's function can be factorized

$$
G^{(+)}\left(\vec{r}, \vec{r}_{s}\right)=\frac{1}{4 \pi^{2}} \int_{0}^{\infty} e^{i \vec{p}_{\| \cdot}^{\left(\vec{r}-\vec{r}_{s}\right)}} g\left(p_{y}, y, y_{s}\right) d^{2} p_{\|},
$$

where $\vec{p}$ is a wave vector with length $k$ and $g\left(p_{y}, y, y_{s}\right)$ is a one-dimensional Green's function perpendicular to the surface. Note, that if $g\left(p_{y}, y, y_{s}\right)$ would be equal to $e^{i p_{y}\left(y-y_{s}\right)} / 2 i p_{y}$ this formula can be converted to Weyls representation of a spherical wave, which in the far zone reduces to

$$
G^{(+)}\left(\vec{r}, \vec{r}_{s}\right)=G_{0}\left(\vec{r}-\vec{r}_{s}\right)=\frac{e^{i k\left|\vec{r}-\vec{r}_{s}\right|}}{4 \pi\left|\vec{r}-\vec{r}_{s}\right|} .
$$

Inserting the Green's function in Eq. (A5) and inverse Fourier transforming, yields

$$
\begin{array}{ll}
\frac{\partial^{2} g}{\partial y^{2}}+p_{y}^{2} g=\delta\left(y-y_{s}\right) & y \geq H \\
\frac{\partial^{2} g}{\partial y^{2}}+\left(p_{y}^{l}\right)^{2} g=\delta\left(y-y_{s}\right) & 0 \leq y<H \\
\frac{\partial^{2} g}{\partial y^{2}}+\left(p_{y}^{s}\right)^{2} g=\delta\left(y-y_{s}\right) & y<0,
\end{array}
$$

where $y=0$ is the position of the substrate-layer interface, $y$ $=H$ is the position of the layer-air interface, $p_{y}^{l}=\sqrt{p_{y}^{2}-\left(k_{c}^{l}\right)^{2}}$ and $p_{y}^{s}=\sqrt{p_{y}^{2}-\left(k_{c}^{s}\right)^{2}}$. The positive sign is taken as $p_{y}$ is also 
positive. The method of variation of parameters can be used to find the particular solution of these inhomogeneous linear second-order differential equations ${ }^{29}$

$$
\begin{array}{cl}
\frac{1}{p_{y}} \int_{-\infty}^{y} \sin \left\{p_{y}(y-t)\right\} \delta\left(t-y_{s}\right) d t \quad y \geq H \\
g_{p}=\frac{1}{p_{y}^{l}} \int_{-\infty}^{y} \sin \left\{p_{y}^{l}(y-t)\right\} \delta\left(t-y_{s}\right) d t \quad 0 \leq y<H \\
\frac{1}{p_{y}^{s}} \int_{-\infty}^{y} \sin \left\{p_{y}^{s}(y-t)\right\} \delta\left(t-y_{s}\right) d t \quad y<0 .
\end{array}
$$

For $y_{s}<0$ this can be reduced to

$$
\begin{array}{cl}
\frac{\sin \left\{p_{y}\left(y-y_{s}\right)\right\}}{p_{y}} & y \geq H \\
g_{p}= & 0 \leq y<H \\
\frac{\sin \left\{p_{y}^{l}\left(y-y_{s}\right)\right\}}{p_{y}^{l}} & \\
0 & y_{s} \leq y<0 \\
p_{y}^{s} & y<y_{s}
\end{array}
$$

for $0 \leq y_{s}<H$

$$
\begin{array}{cl}
\frac{\sin \left\{p_{y}\left(y-y_{s}\right)\right\}}{p_{y}} & y \geq H \\
g_{p}=\frac{\sin \left\{p_{y}^{l}\left(y-y_{s}\right)\right\}}{p_{y}^{l}} & y_{s} \leq y<H \\
0 & y<y_{s}
\end{array}
$$

and for $y_{s} \geq H$

$$
g_{p}=\begin{array}{cc}
\frac{\sin \left\{p_{y}\left(y-y_{s}\right)\right\}}{p_{y}} & y \geq y_{s} \\
0 & y<y_{s} .
\end{array}
$$

The complete solution for the inhomogeneous differential equation can be found by adding the particular solution to the solution of the homogeneous differential equation. The Green's function for $y \rightarrow \infty$ should only have a component away from the surface in the $+y$ direction. For $y \rightarrow-\infty$ it should only have a component in the $-y$ direction. In between it can have both components. Hence, for $y_{s}<0$

$$
\begin{array}{cl}
c_{1} e^{i p_{y} y} & y \geq H \\
c_{1}^{l} e^{i p_{y}^{l} y}+c_{2}^{l} e^{-i p_{y}^{l} y} & 0 \leq y<H \\
g=c_{2}^{s} e^{-i p_{y}^{s} y}+\frac{\sin \left\{p_{y}^{s}\left(y-y_{s}\right)\right\}}{p_{y}^{s}} & y_{s} \leq y<0 \\
c_{2}^{s} e^{-i p_{y}^{s} y} & y<y_{s}
\end{array}
$$

for $0 \leq y_{s}<H$

$$
g=\begin{array}{ll}
c_{1} e^{i p_{y} y} & y \geq H \\
c_{1}^{l} e^{i p_{y}^{l} y}+c_{2}^{l} e^{-i p_{y}^{l} y}+\frac{\sin \left\{p_{y}^{l}\left(y-y_{s}\right)\right\}}{p_{y}^{l}} & y_{s} \leq y<H \\
c_{1}^{l} e^{i p_{y}^{l} y}+c_{2}^{l} e^{-i p_{y}^{l} y} & 0 \leq y<y_{s} \\
c_{2}^{s} e^{-i p_{y}^{s} y} & y<0
\end{array}
$$

and for $y_{s} \geq H$

$$
g=\begin{array}{ll}
c_{1} e^{i p_{y} y} & y \geq y_{s} \\
c_{1} e^{i p_{y} y}-\frac{\sin \left\{p_{y}\left(y-y_{s}\right)\right\}}{p_{y}} & H \leq y<y_{s} \\
c_{1}^{l} e^{i p_{y}^{l} y}+c_{2}^{l} e^{-i p_{y}^{l} y} & 0 \leq y<H \\
c_{2}^{s} e^{-i p_{y}^{s} y} & y<0 .
\end{array}
$$

These solutions and their derivatives to $y$ must be continuous at $y=0$ and $y=H$. In the case considered here $y_{s} \ll y$ and $H$ $\ll y$ hence the one-dimensional Green's function becomes

$$
g=\frac{1}{2 i p_{y}} e^{i p_{y}\left(y-y_{s}\right)} e^{i p_{y} y_{s}} \Psi_{p}\left(y_{s}, H\right),
$$

where $\Psi_{p}\left(y_{s}, H\right)$ describes the wave function in the sample satisfying the boundary conditions for the scattering geometry at hand and for $y_{s}<0$ is given by

$$
\frac{\tau^{l, s}\left(p_{y}\right) \tau^{a, l}\left(p_{y}\right)}{F\left(p_{y}, H\right)} e^{i H\left(p_{y}^{l}-p_{y}\right)-i p_{y}^{s} y_{s}}
$$

for $0 \leq y_{s}<H$ by

$$
\frac{\tau^{a, l}\left(p_{y}\right)\left(\tau^{l, s}\left(p_{y}\right) \cos p_{y}^{l} y_{s}-i \tau^{s, l}\left(p_{y}\right) \sin p_{y}^{l} y_{s}\right)}{F\left(p_{y}, H\right)} e^{i H\left(p_{y}^{l}-p_{y}\right)}
$$

and for $y_{s} \geq H$ by

$$
e^{-i p_{y} y_{s}}+e^{i p_{y}\left(y_{s}-2 H\right)} \frac{\rho^{a, l}\left(p_{y}\right)+\rho^{l, s}\left(p_{y}\right) e^{2 i H p_{y}^{l}}}{F\left(p_{y}, H\right)}
$$

The factor

$$
F\left(p_{y}, H\right)=1+\rho^{a, l}\left(p_{y}\right) \rho^{l, s}\left(p_{y}\right) e^{2 i H p_{y}^{l}}
$$

describes the interference in the layer (note that this factor is 1 if the substrate and the layer have the same potential) and

$$
\tau^{a, l}\left(p_{y}\right)=2 p_{y} /\left(p_{y}+p_{y}^{l}\right)
$$

is the transmission of the wave function traversing the interface from air to layer

$$
\tau^{l, s}\left(p_{y}\right)=2 p_{y}^{l} /\left(p_{y}^{l}+p_{y}^{s}\right)
$$

is the transmission of the wave function traversing the interface from layer to substrate

$$
\tau^{s, l}\left(p_{y}\right)=2 p_{y}^{s} /\left(p_{y}^{l}+p_{y}^{s}\right)
$$

is the transmission of the wave function traversing the interface from substrate to layer 


$$
\rho^{a, l}\left(p_{y}\right)=\left(p_{y}-p_{y}^{l}\right) /\left(p_{y}+p_{y}^{l}\right)
$$

is the reflection coefficient of a wave reflecting at the airlayer interface and

$$
\rho^{l, s}\left(p_{y}\right)=\left(p_{y}^{l}-p_{y}^{s}\right) /\left(p_{y}^{l}+p_{y}^{s}\right)
$$

is the reflection coefficient of the wave reflecting at the layersubstrate interface. The Green's function can be seen as the propagation of a disturbance starting from the source point, $\vec{r}_{s}$ toward the observation point, $\vec{r}$. If the disturbance starts above the sample, there are two possible paths for the disturbance to reach the observation point. One directly and one reflected by the plane surface. The phase difference between these paths obviously depends on the distance to the surface and for small scattering angles and in the far zone is just $2 p_{y}\left(y_{s}-H\right)$. If the disturbance is in the substrate there is only one way to reach the observation point, where the disturbance first propagates through the substrate until it reaches the substrate-layer interface and is partly transmitted, it continuous it path, building phase until it reaches the layer-air interface and is again partly transmitted with a corresponding transmission coefficient. After the transmission the disturbance propagates further to the observation point. Using Eq. (A6) the Green's function becomes

$$
G^{(+)}\left(\vec{r}, \vec{r}_{s}\right)=\frac{1}{4 \pi^{2}} \int_{0}^{\infty} \frac{e^{i \vec{p} \cdot\left(\vec{r}-\vec{r}_{s}\right)}}{2 i p_{y}} e^{i p_{y} y_{s}} \Psi_{p}\left(y_{s}, H\right) d^{2} p_{\|} .
$$

Assuming $e^{i p_{y} y_{s}} \Psi_{p}\left(y_{s}, H\right)$ is constant or a slowly varying function of $\vec{p}$, the Green's function for large distances $(\mid \vec{r}$ $\left.-\vec{r}_{s} \mid k \gg 1\right)$ becomes ${ }^{30}$

$$
G^{(+)}\left(\vec{r}, \vec{r}_{s}\right)=G_{0}\left(\vec{r}-\vec{r}_{s}\right) e^{i p_{y} y_{s}} \Psi_{p}\left(y_{s}, H\right),
$$

where now $\vec{p}=k\left(\vec{r}-\vec{r}_{s}\right) /\left|\vec{r}-\vec{r}_{s}\right|$. When $y_{s}<0$, the condition for a constant or slowly varying value of $e^{i i_{y} y_{s}} \Psi_{p}\left(y_{s}, H\right)$, comes down to $\left|\left(p_{y}-p_{y}^{s}\right) y_{s}\right| \ll 1$ and $\left|\left(p_{y}-p_{y}^{l}\right) H\right| \ll 1$. The first condition is fulfilled when either the sample surface structure is not too high, i.e., $\left|p_{y} y_{s}\right|<1$ or $\left|p_{y} / y_{s}\right|>\left(k_{c}^{s}\right)^{2}$. These conditions start to overlap when $\left|k_{c}^{s} y_{s}\right| \approx 1$ hence as long as $\left|k_{c}^{s} y_{s}\right|$ $\ll 1$ these conditions are fulfilled for all $p_{y}$. This condition will certainly not hold for the nondiffuse component of the scattering as then the integral over $y_{s}$ extends from $-\infty$ to 0 . Similar arguments can be mentioned for the case when 0 $\leq y_{s}<H$. The same result under the same conditions can be derived by using the method of stationary phase for double integrals as discussed by Mandel. ${ }^{8}$
*Present address: BonPhysics Research and Investigations B.V., Laan van Heemstede 38, 3297 AJ, Puttershoek, The Netherlands; victor@bonphysics.nl; http://www.bonphysics.nl

${ }^{1}$ R. Gähler, J. Felber, F. Mezei, and R. Golub, Phys. Rev. A 58, 280 (1998).

${ }^{2}$ H. Rauch, H. Wölwitsch, H. Kaiser, R. Clothier, and S. A. Werner, Phys. Rev. A 53, 902 (1996).

${ }^{3}$ D. A. Pushin, M. Arif, M. G. Huber, and D. G. Cory, Phys. Rev. Lett. 100, 250404 (2008).

${ }^{4}$ V. d. Haan, Phys. Rev. B 77, 104121 (2008).

${ }^{5}$ M. T. Rekveldt, Nucl. Instrum. Methods Phys, Res. B 114, 366 (1996).

${ }^{6}$ M. T. Rekveldt, J. Appl. Crystallogr. 36, 1301 (2003).

${ }^{7}$ V. Holý, U. Pietsch, and T. Baumbach, High-Resolution X-Ray Scattering From Thin Films and Multilayers (Springer-Verlag, Berlin, 1999).

${ }^{8}$ L. Mandel and E. Wolf, Optical Coherence and Quantum Optics, 1st ed. (Cambridge University Press, Cambridge, 1995).

${ }^{9}$ V. F. Sears, Neutron Optics, 1st ed. (Oxford University Press, Oxford, 1989).

${ }^{10}$ Handbook of Mathematical Functions, edited by M. Abramowitz and I. Stegun, National Bureau of Standards, Applied Mathematics Series No. 55, 10th ed. (Dover Publications, New York, 1972).

${ }^{11}$ J. Leckner, Theory of Reflection, 1st ed (Martinus Nijhoff, Dordrecht, 1987).

${ }^{12}$ L. Schiff, Quantum Mechanics (McGraw-Hill, New-York, 1968).

${ }^{13}$ A. Steyerl, Z. Phys. 254, 169 (1972).

${ }^{14}$ S. K. Sinha, E. B. Sirota, S. Garoff, and H. B. Stanley, Phys. Rev. B 38, 2297 (1988).

${ }^{15}$ D. K. G. de Boer, Phys. Rev. B 49, 5817 (1994).
${ }^{16}$ V. de Haan, Coherence Approach to Neutron Propagation in Spin Echo Instruments, 1st ed. (BonPhysics Research and Investigations B.V., Puttershoek, 2007).

${ }^{17}$ M. T. Rekveldt, Physica B 234-236, 1135 (1997).

${ }^{18}$ G. Felcher, S. t. Velthuis, J. Major, A. Dosch, A. Anderson, A. Habicht, and T. Keller, Proc. SPIE 4785, 164 (2002).

${ }^{19}$ J. Major, H. Dosch, G. Felcher, K. Habicht, T. Keller, S. te Velthuis, A. Vorobiev, and M. Wahl, Physica B 336, 8 (2003).

${ }^{20} \mathrm{http} / / /$ www.isis.stfc.ac.uk/instruments /crisp/crisp2466.html

${ }^{21}$ S. K. Sinha, M. Tolan, and A. Gibaud, Phys. Rev. B 57, 2740 (1998).

${ }^{22}$ A. A. van Well, J. Plomp, V. O. de Haan, R. M. Dalgliesh, S. Langridge, W. H. Kraan, W. G. Bouwman, and M. T. Rekveldt, Neutron News 19, 22 (2008).

${ }^{23}$ J. Plomp, V. O. de Haan, A. A. van Well, R. M. Dalgliesh, and S. Langridge, Thin Solid Films 515, 5732 (2007).

${ }^{24} \mathrm{http}$ ://www.isis.stfc.ac.uk/instruments /offspec/offspec3048.html

${ }^{25}$ A. Klein and S. Werner, Rep. Prog. Phys. 46, 259 (1983).

${ }^{26}$ The time-dependent free-particle Green's function for a neutron is given by (Ref. 1) $G_{0}(r, t)=(m / i h t)^{3 / 2} \exp \left(i \pi m r^{2} / h t\right)$. If it is assumed that the wave vector spread $\Delta k$ is sufficiently small (a monochromatic beam), this Green's function reduces to the quoted one.

${ }^{27}$ A. A. Maradudin and D. L. Mills, Phys. Rev. B 11, 1392 (1975).

${ }^{28}$ M. Rauscher, T. Salditt, and H. Spohn, Phys. Rev. B 52, 16855 (1995).

${ }^{29}$ W. E. Boyce and R. D. DiPrima, Elementary Differential Equations and Boundary Value Problems, 4th ed. (Wiley, New York, 1986), pp. 107-185.

${ }^{30}$ D. L. Mills, Phys. Rev. B 12, 4036 (1975). 